\title{
42. PLIOCENE-PLEISTOCENE PALEOCLIMATIC AND PALEOCEANOGRAPHIC HISTORY OF SITE 959, EASTERN EQUATORIAL ATLANTIC OCEAN ${ }^{1}$
}

\author{
Im Chul Shin ${ }^{2}$
}

\begin{abstract}
Pliocene-Pleistocene paleoclimatic and paleoceanographic variables were examined based on the number (in 20 view fields) and relative abundances of calcareous nannofossil $F$. profunda, number of discoasters in 20 view fields, relative abundances of warm- and cool-water indicator calcareous nannofossils, and the species diversity indices $S, H(S)$, and $E$. The early Pliocene is characterized by relatively shallow nutricline (by proxy, thermocline) compared to the late Pliocene as evidenced by lower relative abundances of $F$. profunda. The early Pliocene contains a higher abundance of discoasters, warm-water indicator calcareous nannofossils, and the species diversity indices $S, H(S)$, and $E$ all suggest warm and stable surface-water conditions. The late Pliocene contains the highest relative abundance of $F$. profunda, indicating the deepest nutricline and, by proxy, thermocline. Climatic shift and changes in nutricline/thermocline occurred between 2.82 and 3.03 Ma. The surface-water temperature became cool, the thermocline/nutricline depth became shallower, and the primary productivity began to get higher starting from the early late Pliocene (2.82 Ma) to the latest Pleistocene. These paleoceanographic events are shown by significant changes in both number and relative abundances of F. profunda, relative abundances of warm-water indicator calcareous nannofossils, and by the species diversity indices from the early late Pliocene (upper part of Subzone CN 12a; $2.82 \mathrm{Ma}$ ) through the latest Pleistocene. The early Pleistocene shows greater variations of the relative abundances of $F$. profunda and warm-water nannofossils, suggesting unstable and great fluctuation of surface-water temperature and nutricline/thermocline depth. Another climatic cooling starting approximately $2.4 \mathrm{Ma}$, also shown by the decreasing relative abundances of $F$. profunda, by warmwater indicator nannofossils, and by the species diversity indices for the younger sediment. The middle and late Pleistocene contain the lowest relative abundances of $F$. profunda, suggesting the shallowest thermocline/nutricline.
\end{abstract}

\section{INTRODUCTION}

The purpose of this paper is to investigate the paleoclimatic and paleoceanographic history of the Pliocene to Pleistocene sediment of Hole 959C based on the calcareous nannofossils in the equatorial Atlantic Ocean. Four sites were drilled during Leg 159 (Sites 959-962) on the Marginal Ridge of the Côte d'Ivoire-Ghana Transform Margin (CIGTM) in the eastern Equatorial Atlantic. Four holes were drilled at Site 959: Holes 959A, 959B, 959C, and 959D. Site 959 lies within intermediate and uppermost deep waters. Site 959 is located on a small plateau that extends just north of the top of the Côte d'IvoireGhana Marginal Ridge (CIGMR) on the southern shoulder of the Deep Ivorian Basin (Shipboard Scientific Party, 1996). Pliocene to Pleistocene sediments from Hole 959C are composed of nannofossil ooze with foraminifers. Hole $959 \mathrm{C}$ is at $3^{\circ} 37.669^{\prime} \mathrm{N}, 2^{\circ} 44.116^{\prime} \mathrm{W}$ and has a water depth of $2090 \mathrm{~m}$ (Fig. 1). Hole 959C was drilled using advanced hydraulic piston coring (APC), until refusal at $179.6 \mathrm{mbsf}$.

Core recovery from Hole 959C was complete, and contains wellpreserved abundant calcareous nannofossils. Therefore, Hole 959C is an ideal site for the study of calcareous nannofossil paleoceanography.

\section{METHODS}

Conventionally, the smear slide method is used by calcareous nannofossil paleontologists for the paleoceanography and quantitative study of calcareous nannofossils. However, size fractionation on smear slides has been observed as a result of toothpick action on a slide (Wei, 1988). Beaufort (1991; p. 415) stressed that "a count of

${ }^{1}$ Mascle, J., Lohmann, G.P., and Moullade, M. (Eds.), 1998. Proc. ODP, Sci. Results, 159: College Station, TX (Ocean Drilling Program).

${ }^{2}$ Department of Geology, University of Nebraska-Lincoln, Lincoln, NE 685880340, U.S.A. (Present address: Korea Ocean Research \& Development Institute, Ansan, P.O. Box 29, Seoul 425-600 Korea.) icshin@sari.kordi.re.kr relative abundance using smear slides is accurate only for species of similar size unless a large number of view fields are examined." To avoid the size fractionation of calcareous nannofossils during the preparation of smear slides, the settling method developed by Beaufort (1991) and Ehrendorfer (1993) is used for the study of paleoceanography.

Neogene zonation of Okada and Bukry (1980) was used for the biostratigraphy. The age assignments of marker species were followed Berggren et al. $(1985,1995)$. Neogene (Pliocene to Pleistocene) samples totaling 371 from interval $159-959 \mathrm{C}-1 \mathrm{H}$ to $8 \mathrm{H}$ were selected, using 20-cm sampling intervals. Calcareous nannofossil Florisphaera profunda was counted in 20 view fields at $1000 \times$ mag-

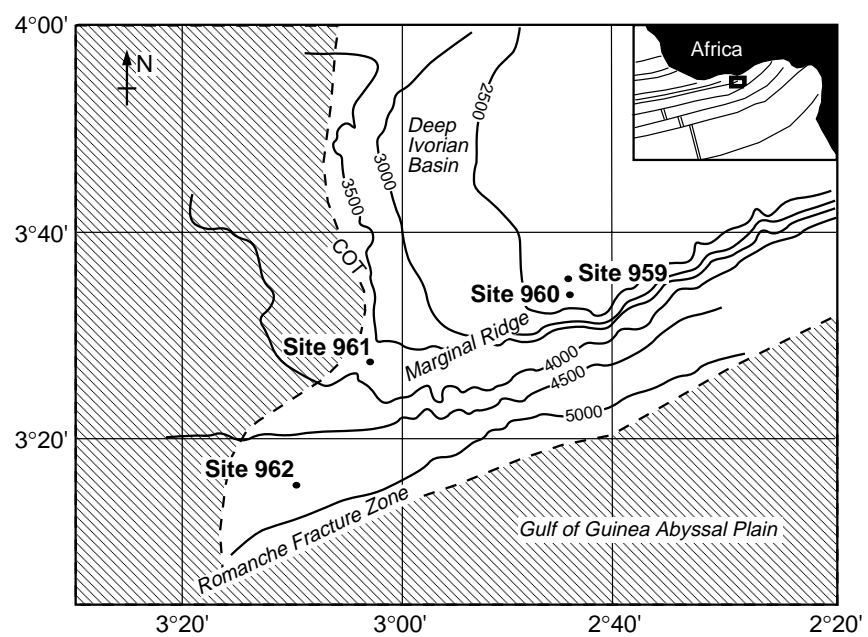

Figure 1. Location map of Site 959 and the other Leg 159 sites. 
nification (Table 1). Relative abundances of calcareous nannoplankton species per section (1.5-m intervals) are reported based on 500 countings from the settled slide (Shin et al., Chap. 39, this volume). Relative abundances of $F$. profunda in each section (1.5-m intervals) are used to study the nutricline/thermocline depth variations.

The species diversity indices of calcareous nannofossils were calculated based on the percentage data in terms of species richness, $S$, Shannon-Wiener information function $H(S)$, and equitability $(E)$ (Table 2). Richness was estimated by the number of species per settled slide, using a count of 500 specimens. The Shannon-Wiener information function, derived from information theory, is defined as $H(S)=$ $-\Sigma P_{i} \times \ln P_{i}$, where $S$ is the number of species in the sample and $P_{i}$ is the proportion of the $i_{\text {th }}$ species in the sample. One advantage of this information function is the minimization of problems related to sample size. This information function is little affected by rare and extremely abundant species and is affected mostly by species with common abundances (Gibson and Buzas, 1973). Theoretically, the maximum value of $H(S)$ occurs when all species are equally distributed.

Equitability $(E)$, used here, is the Buzas and Gibson equation (1969). It is defined as $E=e^{H(S)} / S$ where $H(S)$ is the Shannon-Wiener index for the sample and $S$ is the number of species in the sample. Values of $E$ are always less than one and measure how far the sample departs from complete equitability. When all species are equally distributed, $E=1.0$. A low value for $E$ indicates greater deviation from a sample of equally proportioned species. In this study, the species diversity indices were used as a proxy measure of the surface-water temperatures and the stability of the surface-water mass conditions.

The variations in relative abundance of cool- and warm-water calcareous nannofossil species also were used as a measure of surfacewater paleotemperature conditions. The cool-water indicator calcareous nannofossil species used in this study are Coccolithus pelagicus, Discoaster asymmetricus, Emiliania huxleyi, and Gephyrocapsa caribbeanica. The warm-water indicator nannofossil species used in this study are Calcidiscus leptoporus, different species of Discoaster (except D. asymmetricus), Florisphaera profunda, Gephyrocapsa oceanica, Sphenolithus abies, S. neoabies, and Umbellosphaera irregularis. The following references were used for the selection of warmand cool-water indicator calcareous nannofossils (McIntyre et al., 1970; Okada and Honjo, 1973, 1975; Bukry, 1978; Decima et al., 1978; Haq et al., 1977; Haq and Lohmann, 1976; Honjo, 1977; Schneidermann, 1977; Okada and McIntyre, 1979; Backman and Shackleton, 1983; Manivit, 1989; Chepstow-Lusty et al., 1989; Rio et al., 1990; Spaulding, 1991). The number of Discoaster in 20 view fields at $1000 \times$ magnification was counted to know the surface-water temperature variations (Table 2).

\section{RESULTS AND DISCUSSION Paleoclimatic and Paleoceanographic History of Hole 959C}

The number of $F$. profunda in 20 view fields in each 20 -cm interval ranges from 74 to 1664 individuals (Fig. 2; Table 1). Figures 3 and 4 show the downcore distribution patterns of the numbers and relative abundances of $F$. profunda in each $1.5-\mathrm{m}$ interval ( 1 sample/section). Florisphaera profunda was first described from the Pacific Ocean by Okada and Honjo (1973). This species is easy to recognize and is well preserved in oceanic sediments (Okada and Honjo, 1973). It lives only in the lower photic layer (150-200 m) and prefers low light and high nutrients (Honjo, 1977; Okada and Honjo, 1973; Honjo and Okada, 1974; Molfino and McIntyre, 1990a). The variations of this species were interpreted as changes in water depth, nutricline (by proxy, thermocline) depth, and water transparency and turbidity (Okada and Honjo, 1973; Honjo and Okada, 1974; Honjo, 1977; Okada and McIntyre, 1977, 1979; Reid, 1980; Okada, 1984, 1990; Molfino and McIntyre, 1990a, 1990b; Ahagon et al., 1993).

Climate control of nutricline/thermocline depth can be monitored by variations of $F$. profunda (Molfino and McIntyre, 1990a, 1990b).
Molfino and McIntyre (1990a, 1990b) suggested that the high relative abundances (\%) of $F$. profunda indicate a deep nutricline and warm surface-water temperatures associated with wind strength, divergence, and primary productivity minima. A deep nutricline means the upper euphotic zone is nutrient depleted. Nutricline depth is defined by a level of $\mathrm{PO}_{4}$ of $1.0 \mu \mathrm{mol} / \mathrm{liter}$, and it varies between 50 and $200 \mathrm{~m}$ (Molfino and McIntyre, 1990b). Therefore, there is a close relationship between the relative abundances of $F$. profunda and the nutrient content in the euphotic water column (Molfino and McIntyre, 1990a, 1990b; Ahagon et al., 1993). When the nutricline (by proxy, thermocline) becomes deep, the production of $F$. profunda in the upper euphotic layer is greater than the production of coccolithophorids (Molfino and McIntyre, 1990a, 1990b). On the other hand, lower relative abundances of $F$. profunda are associated with shallow nutricline/thermocline and low sea-surface temperatures because of wind strength, divergence, and primary productivity maxima (Molfino and McIntyre, 1990a, 1990b).

The early Pliocene in Hole $959 \mathrm{C}$ is characterized by lower relative abundances of $F$. profunda compared to late Pliocene (Fig. 4). This suggests that the nutricline/thermocline is relatively shallower in the early Pliocene than that in the late Pliocene. Figure 5 shows the number of Discoasters in 20 view fields. Discoaster spp. have an ecological preference for warm waters (Haq and Lohmann, 1976; Haq et al., 1977; Backman and Shackleton, 1983) and are resistant to dissolution (Bukry, 1971; Roth, 1973; Lohmann and Carlson, 1981; Manivit, 1989). Total Discoaster abundance is sensitive to temperature changes (Haq and Lohmann, 1976; Chepstow-Lusty et al., 1989). The plot of the number of Discoaster spp. does not show exactly the same trend as the relative abundances and number of $F$. profunda (Figs. 3-5). However, the number of Discoaster spp. shows a rough decreasing trend from the early Pliocene through the latest Pliocene, suggesting that surface-water temperatures became cooler from the early Pliocene through the latest Pliocene (Fig. 5). The Discoaster accumulation rate during the early Pliocene is four times higher than the late Pliocene in the North Atlantic according to Backman et al., (1986). They interpreted this as warmer surface water during the early Pliocene compared to the late Pliocene. This study also shows significantly higher numbers of Discoaster during the early Pliocene than the late Pliocene. Higher numbers of Discoaster, higher relative abundances of warm-water calcareous nannofossils, and greater values and smaller fluctuations of species diversity indices $S, H(S)$, and $E$ during the early Pliocene suggest warm and stable surface-water conditions (Figs. 5-7).

Backman and Pestiaux (1987) demonstrated that surface water shows continuous decreasing temperature from 3.5 Ma to the younger sediment based on Discoaster accumulation rate in the North Atlantic. This study shows a decreasing trend of the number of Discoaster spp. from 3.65 Ma to the younger sediment (Fig. 5).

Calcareous nannofossil richness declines during climatic cooling in the Cenozoic (Wise, 1988). Huber and Watkins (1992) also interpreted the lower number of species as an indication of cool climate during the Maastrichtian. Honjo and Okada (1974) showed that modern day coccolith richness is highest in the equatorial zone. Winter et al. (1979) demonstrated that high species richness in modern nannoplankton communities is correlated with high water temperatures, and low diversity is characteristic of relatively cool periods. Haq (1971) showed greater calcareous nannofossil species richness at climatic warming during the Paleogene period. Schneidermann (1977) reported richness decreases toward polar regions. Winter et al. (1979) found higher richness can correlate with high water temperature (summer) and low richness in cool winter period. Okada and McIntyre (1979) also reported higher diversity in late summer or autumn in the North Atlantic Ocean. From this study, the species richness (S) shows moderate positive correlation to the sum of warm $(r=0.65)$ and cool $(\mathrm{r}=-0.55)$ water indicator calcareous nannofossils. This suggests that higher richness (S) occurs in warm water. Pliocene sediments have higher richness values than those of the cool Pleistocene (Fig. 7). Manivit (1989) also reported lower species richness in the 
Table 1. List of the number of calcareous nannofossil species $F$. profunda and its biostratigraphic zone in each 20 -cm interval.

\begin{tabular}{|c|c|c|c|c|c|c|}
\hline $\begin{array}{l}\text { Core, section, } \\
\text { interval }(\mathrm{cm})\end{array}$ & $\begin{array}{l}\text { Depth } \\
\text { (mbsf) }\end{array}$ & F. profunda & Zone & $\begin{array}{l}\text { Core, section, } \\
\text { interval }(\mathrm{cm})\end{array}$ & $\begin{array}{l}\text { Depth } \\
\text { (mbsf) }\end{array}$ & F. profunda \\
\hline $159-959 \mathrm{C}-$ & & & & $3 \mathrm{H}-4,40-42.5$ & 16.71 & 210 \\
\hline $1 \mathrm{H}-1,0-2.5$ & 0.01 & 393 & CN15-CN14b & $3 \mathrm{H}-4,60-62.5$ & 16.91 & 319 \\
\hline $1 \mathrm{H}-1,20-22.5$ & 0.21 & 410 & CN15-CN14b & $3 \mathrm{H}-4,80-82.5$ & 17.11 & 371 \\
\hline $1 \mathrm{H}-1,40-42.5$ & 0.41 & 681 & CN15-CN14b & $3 \mathrm{H}-4,100-102.5$ & 17.31 & 398 \\
\hline $1 \mathrm{H}-1,60-62.5$ & 0.61 & 175 & CN15-CN14b & $3 \mathrm{H}-4,120-122.5$ & 17.51 & 339 \\
\hline $1 \mathrm{H}-1,80-82.5$ & 0.81 & 308 & CN15-CN14b & $3 \mathrm{H}-4,140-142.5$ & 17.71 & 173 \\
\hline $1 \mathrm{H}-1,100-102.5$ & 1.01 & 328 & CN15-CN14b & $3 \mathrm{H}-5,0-2.5$ & 17.81 & 536 \\
\hline $1 \mathrm{H}-1,120-122.5$ & 1.21 & 238 & $\mathrm{CN} 15-\mathrm{CN} 14 \mathrm{~b}$ & $3 \mathrm{H}-5,20-22.5$ & 18.01 & 532 \\
\hline $1 \mathrm{H}-1,140-142.5$ & 1.41 & 220 & CN15-CN14b & $3 \mathrm{H}-5,40-42.5$ & 18.21 & 541 \\
\hline $1 \mathrm{H}-2,0-2.5$ & 1.51 & 298 & CN15-CN14b & $3 \mathrm{H}-5,60-62.5$ & 18.41 & 508 \\
\hline $1 \mathrm{H}-2,20-22.5$ & 1.71 & 272 & CN15-CN14b & $3 \mathrm{H}-5,80-82.5$ & 18.61 & 416 \\
\hline $1 \mathrm{H}-2,40-42.5$ & 1.91 & 448 & CN15-CN14b & $3 \mathrm{H}-5,100-102.5$ & 18.81 & 373 \\
\hline $1 \mathrm{H}-2,60-62.5$ & 2.11 & 213 & CN15-CN14b & $3 \mathrm{H}-5,120-122.5$ & 19.01 & 84 \\
\hline $2 \mathrm{H}-1,0-2.5$ & 2.31 & 178 & $\mathrm{CN} 15-\mathrm{CN} 14 \mathrm{~b}$ & $3 \mathrm{H}-5,140-142.5$ & 19.21 & 348 \\
\hline $2 \mathrm{H}-1,20-22.5$ & 2.51 & 265 & $\mathrm{CN} 15-\mathrm{CN} 14 \mathrm{~b}$ & $3 \mathrm{H}-6,0-2.5$ & 19.31 & 380 \\
\hline $2 \mathrm{H}-1,40-42.5$ & 2.71 & 166 & $\mathrm{CN} 15-\mathrm{CN} 14 \mathrm{~b}$ & $3 \mathrm{H}-6,20-22.5$ & 19.51 & 293 \\
\hline $2 \mathrm{H}-1,60-62.5$ & 2.91 & 178 & CN15-CN14b & $3 \mathrm{H}-6,40-42.5$ & 19.71 & 256 \\
\hline $2 \mathrm{H}-1,80-82.5$ & 3.11 & 178 & CN15-CN14b & $3 \mathrm{H}-6,80-82.5$ & 20.11 & 487 \\
\hline $2 \mathrm{H}-1,100-102.5$ & 3.31 & 212 & CN15-CN14b & $3 \mathrm{H}-6,100-102.5$ & 20.31 & 350 \\
\hline $2 \mathrm{H}-1,120-122.5$ & 3.51 & 170 & $\mathrm{CN} 15-\mathrm{CN} 14 \mathrm{~b}$ & $3 \mathrm{H}-6,120-122.5$ & 20.51 & 258 \\
\hline $2 \mathrm{H}-1,140-142.5$ & 3.71 & 170 & $\mathrm{CN} 15-\mathrm{CN} 14 \mathrm{~b}$ & $3 \mathrm{H}-6,140-142.5$ & 20.71 & 283 \\
\hline $2 \mathrm{H}-2,0-2.5$ & 3.81 & 117 & CN15-CN14b & $3 \mathrm{H}-7,0-2.5$ & 20.81 & 457 \\
\hline $2 \mathrm{H}-2,20-22.5$ & 4.01 & 111 & CN15-CN14b & $3 \mathrm{H}-7,20-22.5$ & 21.01 & 240 \\
\hline $2 \mathrm{H}-2,40-42.5$ & 4.21 & 118 & CN15-CN14b & $3 \mathrm{H}-7,40-42.5$ & 21.21 & 227 \\
\hline $2 \mathrm{H}-2,60-62.5$ & 4.41 & 169 & CN15-CN14b & $4 \mathrm{H}-1,0-2.5$ & 21.31 & 74 \\
\hline $2 \mathrm{H}-2,80-82.5$ & 4.61 & 107 & $\mathrm{CN} 15-\mathrm{CN} 14 \mathrm{~b}$ & $4 \mathrm{H}-1,20-22.5$ & 21.51 & 293 \\
\hline $2 \mathrm{H}-2,100-102.5$ & 4.81 & 159 & $\mathrm{CN} 14 \mathrm{a}$ & $4 \mathrm{H}-1,40-42.5$ & 21.71 & 270 \\
\hline $2 \mathrm{H}-2,120-122.5$ & 5.01 & 269 & $\mathrm{CN} 14 \mathrm{a}$ & $4 \mathrm{H}-1,60-62.5$ & 21.91 & 65 \\
\hline $2 \mathrm{H}-2,140-142.5$ & 5.21 & 85 & CN14a & $4 \mathrm{H}-1,80-82.5$ & 22.11 & 277 \\
\hline $2 \mathrm{H}-3,0-2.5$ & 5.31 & 80 & CN14a & $4 \mathrm{H}-1,100-102.5$ & 22.31 & 160 \\
\hline $2 \mathrm{H}-3,20-22.5$ & 5.51 & 258 & $\mathrm{CN} 14 \mathrm{a}$ & $4 \mathrm{H}-1,120-122.5$ & 22.51 & 138 \\
\hline $2 \mathrm{H}-3,40-42.5$ & 5.71 & 270 & $\mathrm{CN} 14 \mathrm{a}$ & $4 \mathrm{H}-1,140-142.5$ & 22.71 & 122 \\
\hline $2 \mathrm{H}-3,60-62.5$ & 5.91 & 170 & $\mathrm{CN} 14 \mathrm{a}$ & $4 \mathrm{H}-2,0-2.5$ & 22.81 & 177 \\
\hline $2 \mathrm{H}-3,80-82.5$ & 6.11 & 257 & $\mathrm{CN} 14 \mathrm{a}$ & $4 \mathrm{H}-2,20-22.5$ & 23.01 & 181 \\
\hline $2 \mathrm{H}-3,100-102.5$ & 6.31 & 307 & CN14a & $4 \mathrm{H}-2,40-42.5$ & 23.21 & 333 \\
\hline $2 \mathrm{H}-3,120-122.5$ & 6.51 & 371 & CN14a & $4 \mathrm{H}-2,60-62.5$ & 23.41 & 169 \\
\hline $2 \mathrm{H}-3,140-142.5$ & 6.71 & 151 & $\mathrm{CN} 14 \mathrm{a}$ & $4 \mathrm{H}-2,80-82.5$ & 23.61 & 240 \\
\hline $2 \mathrm{H}-4,0-2.5$ & 6.81 & 254 & $\mathrm{CN} 14 \mathrm{a}$ & $4 \mathrm{H}-2,100-102.5$ & 23.81 & 125 \\
\hline $2 \mathrm{H}-4,20-22.5$ & 7.01 & 198 & $\mathrm{CN} 14 \mathrm{a}$ & $4 \mathrm{H}-2,120-122.5$ & 24.01 & 218 \\
\hline $2 \mathrm{H}-4,40-42.5$ & 7.21 & 206 & $\mathrm{CN} 14 \mathrm{a}$ & $4 \mathrm{H}-2,140-142.5$ & 24.21 & 390 \\
\hline $2 \mathrm{H}-4,60-62.5$ & 7.41 & 197 & CN14a & $4 \mathrm{H}-3,0-2.5$ & 24.31 & 370 \\
\hline $2 \mathrm{H}-4,80-82.5$ & 7.61 & 361 & CN14a & $4 \mathrm{H}-3,40-42.5$ & 24.71 & 472 \\
\hline $2 \mathrm{H}-4,100-102.5$ & 7.81 & 363 & $\mathrm{CN} 14 \mathrm{a}$ & $4 \mathrm{H}-3,60-62.5$ & 24.91 & 395 \\
\hline $2 \mathrm{H}-4,120-122.5$ & 8.01 & 380 & $\mathrm{CN} 14 \mathrm{a}$ & $4 \mathrm{H}-3,80-82.5$ & 25.11 & 413 \\
\hline $2 \mathrm{H}-4,140-142.5$ & 8.21 & 260 & $\mathrm{CN} 14 \mathrm{a}$ & $4 \mathrm{H}-3,100-102.5$ & 25.31 & 395 \\
\hline $2 \mathrm{H}-5,0-2.5$ & 8.31 & 233 & $\mathrm{CN} 14 \mathrm{a}$ & $4 \mathrm{H}-3,120-122.5$ & 25.51 & 274 \\
\hline $2 \mathrm{H}-5,20-22.5$ & 8.51 & 360 & CN14a & $4 \mathrm{H}-3,140-142.5$ & 25.71 & 182 \\
\hline $2 \mathrm{H}-5,40-42.5$ & 8.71 & 314 & $\mathrm{CN} 14 \mathrm{a}$ & $4 \mathrm{H}-4,0-2.5$ & 25.81 & 231 \\
\hline $2 \mathrm{H}-5,60-62.5$ & 8.91 & 296 & $\mathrm{CN} 14 \mathrm{a}$ & $4 \mathrm{H}-4,20-22.5$ & 26.01 & 345 \\
\hline $2 \mathrm{H}-5,80-82.5$ & 9.11 & 960 & CN14a & $4 \mathrm{H}-4,40-42.5$ & 26.21 & 190 \\
\hline $2 \mathrm{H}-5,100-102.5$ & 9.31 & 373 & $\mathrm{CN} 14 \mathrm{a}$ & $4 \mathrm{H}-4,60-62.5$ & 26.41 & 288 \\
\hline $2 \mathrm{H}-5,120-122.5$ & 9.51 & 386 & CN14a & $4 \mathrm{H}-4,80-82.5$ & $\begin{array}{l}20.41 \\
26.61\end{array}$ & 487 \\
\hline $2 \mathrm{H}-5,140-142.5$ & 9.71 & 151 & CN14a & $4 \mathrm{H}-4,100-102.5$ & $\begin{array}{l}20.01 \\
26.81\end{array}$ & $\begin{array}{l}401 \\
228\end{array}$ \\
\hline $2 \mathrm{H}-6,0-2.5$ & 9.81 & 316 & CN14a & $4 \mathrm{H}-4,120-122.5$ & 27.01 & 418 \\
\hline $2 \mathrm{H}-6,20-22.5$ & 10.01 & 145 & CN14a & $4 \mathrm{H}-4,140-142.5$ & 27.21 & 283 \\
\hline $2 \mathrm{H}-6,40-42.5$ & 10.21 & 233 & CN14a & $4 \mathrm{H}-5,0-2.5$ & 27.31 & $\begin{array}{l}203 \\
269\end{array}$ \\
\hline $2 \mathrm{H}-6,60-62.5$ & 10.41 & 265 & $\mathrm{CN} 14 \mathrm{a}$ & $4 \mathrm{H}-5,20-22.5$ & 27.51 & 225 \\
\hline $2 \mathrm{H}-6,80-82.5$ & 10.61 & 356 & CN14a & $4 \mathrm{H}-5,40-42.5$ & 27.71 & 413 \\
\hline $2 \mathrm{H}-6,100-102.5$ & 10.81 & 385 & CN14a & $4 \mathrm{H}-5,60-62.5$ & 27.91 & 432 \\
\hline $2 \mathrm{H}-6,120-122.5$ & 11.01 & 165 & CN14a & $4 \mathrm{H}-5,80-82.5$ & 28.11 & 264 \\
\hline $2 \mathrm{H}-6,140-142.5$ & 11.21 & 255 & $\mathrm{CN} 14 \mathrm{a}$ & $4 \mathrm{H}-5,100-102.5$ & 28.31 & 265 \\
\hline $2 \mathrm{H}-7,0-2.5$ & 11.31 & 254 & $\mathrm{CN} 14 \mathrm{a}$ & $4 \mathrm{H}-5,120-122.5$ & 28.51 & 440 \\
\hline $2 \mathrm{H}-7,20-22.5$ & 11.51 & 291 & CN14a & $4 \mathrm{H}-5,140-142.5$ & 28.71 & 511 \\
\hline $2 \mathrm{H}-7,40-42.5$ & 11.71 & 416 & CN14a & $4 \mathrm{H}-6,0-2.5$ & 28.81 & 505 \\
\hline $2 \mathrm{H}-7,60-62.5$ & 11.80 & 233 & CN14a & $4 \mathrm{H}-6,20-22.5$ & 29.01 & 196 \\
\hline $3 \mathrm{H}-1,0-2.5$ & 11.81 & 416 & $\mathrm{CN} 14 \mathrm{a}$ & $4 \mathrm{H}-6,40-42.5$ & 29.21 & 472 \\
\hline $3 \mathrm{H}-1,20-22.5$ & 12.01 & 251 & $\mathrm{CN} 14 \mathrm{a}$ & $4 \mathrm{H}-6,60-62.5$ & 29.41 & 358 \\
\hline $3 \mathrm{H}-1,40-42.5$ & 12.21 & 392 & $\mathrm{CN} 14 \mathrm{a}$ & $4 \mathrm{H}-6,80-82.5$ & 29.61 & 507 \\
\hline $3 \mathrm{H}-1,60-62.5$ & 12.41 & 488 & $\mathrm{CN} 14 \mathrm{a}$ & $4 \mathrm{H}-6,100-102.5$ & 29.81 & 531 \\
\hline $3 \mathrm{H}-1,80-82.5$ & 12.61 & 219 & $\mathrm{CN} 14 \mathrm{a}$ & $4 \mathrm{H}-6,120-122.5$ & 30.01 & 592 \\
\hline $3 \mathrm{H}-1,100-102.5$ & 12.81 & 218 & CN13a & $4 \mathrm{H}-6,140-142.5$ & 30.21 & 490 \\
\hline $3 \mathrm{H}-1,120-122.5$ & 13.01 & 293 & $\mathrm{CN} 13 \mathrm{a}$ & $4 \mathrm{H}-7,0-2.5$ & 30.31 & 300 \\
\hline $3 \mathrm{H}-1,140-142.5$ & 13.21 & 220 & CN13a & $4 \mathrm{H}-7,20-22.5$ & 30.51 & 208 \\
\hline $3 \mathrm{H}-2,0-2.5$ & 13.31 & 183 & $\mathrm{CN} 13 \mathrm{a}$ & $4 \mathrm{H}-7,40-42.5$ & 30.71 & 329 \\
\hline $3 \mathrm{H}-2,20-22.5$ & 13.51 & 188 & $\mathrm{CN} 13 \mathrm{a}$ & $4 \mathrm{H}-7,60-62.5$ & 30.80 & 522 \\
\hline $3 \mathrm{H}-2,40-42.5$ & 13.71 & 75 & $\mathrm{CN} 13 \mathrm{a}$ & $5 \mathrm{H}-1,0-2.5$ & 30.81 & 523 \\
\hline $3 \mathrm{H}-2,60-62.5$ & 13.91 & 126 & CN13a & $5 \mathrm{H}-1,20-22.5$ & 31.01 & 448 \\
\hline $3 \mathrm{H}-2,80-82.5$ & 14.11 & 257 & $\mathrm{CN} 13 \mathrm{a}$ & $5 \mathrm{H}-1,40-42.5$ & 31.21 & 531 \\
\hline $3 \mathrm{H}-2,100-102.5$ & 14.31 & 235 & $\mathrm{CN} 13 \mathrm{a}$ & $5 \mathrm{H}-1,60-62.5$ & 31.41 & 218 \\
\hline $3 \mathrm{H}-2,120-122.5$ & 14.51 & 271 & CN13a & $5 \mathrm{H}-1,80-82.5$ & 31.61 & 871 \\
\hline $3 \mathrm{H}-2,140-142.5$ & 14.71 & 309 & $\mathrm{CN} 13 \mathrm{a}$ & $5 \mathrm{H}-1,100-102.5$ & 31.81 & 336 \\
\hline $3 \mathrm{H}-3,0-2.5$ & 14.81 & 201 & $\mathrm{CN} 13 \mathrm{a}$ & $5 \mathrm{H}-1,120-122.5$ & 32.01 & 731 \\
\hline $3 \mathrm{H}-3,20-22.5$ & 15.01 & 322 & CN13b & $5 \mathrm{H}-1,140-142.5$ & 32.21 & 441 \\
\hline $3 \mathrm{H}-3,40-42.5$ & 15.21 & 338 & CN13b & $5 \mathrm{H}-2,0-2.5$ & 32.31 & 670 \\
\hline $3 \mathrm{H}-3,60-62.5$ & 15.41 & 177 & CN13b & $5 \mathrm{H}-2,20-22.5$ & 32.51 & 790 \\
\hline $3 \mathrm{H}-3,80-82.5$ & 15.61 & 424 & CN13b & $5 \mathrm{H}-2,40-42.5$ & 32.71 & 990 \\
\hline $3 \mathrm{H}-3,100-102.5$ & 15.81 & 388 & CN13b & $5 \mathrm{H}-2,60-62.5$ & 32.91 & 333 \\
\hline $3 \mathrm{H}-3,120-122.5$ & 16.01 & 317 & CN13b & $5 \mathrm{H}-2,80-82.5$ & 33.11 & 752 \\
\hline $3 \mathrm{H}-3,140-142.5$ & 16.21 & 586 & CN13b & $5 \mathrm{H}-2,100-102.5$ & 33.31 & 517 \\
\hline $3 \mathrm{H}-4,0-2.5$ & 16.31 & 270 & $\mathrm{CN} 13 \mathrm{~b}$ & $5 \mathrm{H}-2,120-122.5$ & 33.51 & 832 \\
\hline $3 \mathrm{H}-4,20-22.5$ & 16.51 & 372 & $\mathrm{CN} 13 \mathrm{~b}$ & & & \\
\hline
\end{tabular}


Table 1 (continued).

\begin{tabular}{|c|c|c|c|}
\hline $\begin{array}{l}\text { Core, section, } \\
\text { interval }(\mathrm{cm})\end{array}$ & $\begin{array}{l}\text { Depth } \\
\text { (mbsf) }\end{array}$ & F. profunda & Zone \\
\hline \multicolumn{4}{|l|}{$159-959 \mathrm{C}-$} \\
\hline $5 \mathrm{H}-2,140-142.5$ & 33.71 & 975 & $\mathrm{CN} 12 \mathrm{c}$ \\
\hline $5 \mathrm{H}-3,0-2.5$ & 34.01 & 942 & $\mathrm{CN} 12 \mathrm{c}$ \\
\hline $5 \mathrm{H}-3,20-22.5$ & 34.01 & 942 & $\mathrm{CN} 12 \mathrm{c}$ \\
\hline $5 \mathrm{H}-3,40-42.5$ & 34.21 & 693 & $\mathrm{CN} 12 \mathrm{c}$ \\
\hline $5 \mathrm{H}-3,60-62.5$ & 34.41 & 725 & $\mathrm{CN} 12 \mathrm{c}$ \\
\hline $5 \mathrm{H}-3,80-82.5$ & 34.61 & 1081 & $\mathrm{CN} 12 \mathrm{c}$ \\
\hline $5 \mathrm{H}-3,100-102.5$ & 34.81 & 1065 & $\mathrm{CN} 12 \mathrm{c}$ \\
\hline $5 \mathrm{H}-3,120-122.5$ & 35.01 & 196 & $\mathrm{CN} 12 \mathrm{c}$ \\
\hline $5 \mathrm{H}-3,140-142.5$ & 35.21 & 583 & $\mathrm{CN} 12 \mathrm{c}$ \\
\hline $5 \mathrm{H}-4,0-2.5$ & 35.31 & 748 & $\mathrm{CN} 12 \mathrm{c}$ \\
\hline $5 \mathrm{H}-4,20-22.5$ & 35.51 & 687 & $\mathrm{CN} 12 \mathrm{c}$ \\
\hline $5 \mathrm{H}-4,40-42.5$ & 35.71 & 626 & $\mathrm{CN} 12 \mathrm{c}$ \\
\hline $5 \mathrm{H}-4,60-62.5$ & 35.91 & 394 & $\mathrm{CN} 12 \mathrm{c}$ \\
\hline $5 \mathrm{H}-4,80-82.5$ & 36.11 & 953 & $\mathrm{CN} 12 \mathrm{c}$ \\
\hline $5 \mathrm{H}-4,100-102.5$ & 36.31 & 827 & $\mathrm{CN} 12 \mathrm{c}$ \\
\hline $5 \mathrm{H}-4,120-122.5$ & 36.51 & 653 & $\mathrm{CN} 12 \mathrm{c}$ \\
\hline $5 \mathrm{H}-4,140-142.5$ & 36.71 & 607 & $\mathrm{CN} 12 \mathrm{c}$ \\
\hline $5 \mathrm{H}-5,0-2.5$ & 36.81 & 551 & $\mathrm{CN} 12 \mathrm{c}$ \\
\hline $5 \mathrm{H}-5,20-22.5$ & 37.01 & 853 & $\mathrm{CN} 12 \mathrm{c}$ \\
\hline $5 \mathrm{H}-5,40-42.5$ & 37.21 & 528 & $\mathrm{CN} 12 \mathrm{c}$ \\
\hline $5 \mathrm{H}-5,60-62.5$ & 37.41 & 525 & $\mathrm{CN} 12 \mathrm{c}$ \\
\hline $5 \mathrm{H}-5,80-82.5$ & 37.61 & 614 & $\mathrm{CN} 12 \mathrm{c}$ \\
\hline $5 \mathrm{H}-5,100-102.5$ & 37.81 & 820 & $\mathrm{CN} 12 \mathrm{c}$ \\
\hline $5 \mathrm{H}-5,12 \mathrm{O}-122.5$ & 38.01 & 226 & $\mathrm{CN} 12 \mathrm{c}$ \\
\hline $5 \mathrm{H}-5,140-142.5$ & 38.21 & 445 & $\mathrm{CN} 12 \mathrm{c}$ \\
\hline $5 \mathrm{H}-6,0-2.5$ & 38.31 & 565 & $\mathrm{CN} 12 \mathrm{c}$ \\
\hline $5 \mathrm{H}-6,20-22.5$ & 38.51 & 681 & $\mathrm{CN} 12 \mathrm{c}$ \\
\hline $5 \mathrm{H}-6,40-42.5$ & 38.71 & 1100 & $\mathrm{CN} 12 \mathrm{c}$ \\
\hline $5 \mathrm{H}-6,60-62.5$ & 38.91 & 603 & $\mathrm{CN} 12 \mathrm{c}$ \\
\hline $5 \mathrm{H}-6,80-82.5$ & 39.11 & 890 & $\mathrm{CN} 12 \mathrm{c}$ \\
\hline $5 \mathrm{H}-6,100-102.5$ & 39.31 & 745 & $\mathrm{CN} 12 \mathrm{c}$ \\
\hline $5 \mathrm{H}-6,120-122.5$ & 39.51 & 745 & $\mathrm{CN} 12 \mathrm{c}$ \\
\hline $5 \mathrm{H}-6,140-142.5$ & 39.71 & 421 & $\mathrm{CN} 12 \mathrm{c}$ \\
\hline $5 \mathrm{H}-7,0-2.5$ & 39.81 & 292 & $\mathrm{CN} 12 \mathrm{c}$ \\
\hline $5 \mathrm{H}-7,20-22.5$ & 40.01 & 606 & $\mathrm{CN} 12 \mathrm{c}$ \\
\hline $5 \mathrm{H}-7,40-42.5$ & 40.21 & 650 & $\mathrm{CN} 12 \mathrm{c}$ \\
\hline $5 \mathrm{H}-7,60-62.5$ & 40.30 & 558 & $\mathrm{CN} 12 \mathrm{~b}$ \\
\hline $6 \mathrm{H}-1,0-2.5$ & 40.31 & 850 & $\mathrm{CN} 12 \mathrm{~b}$ \\
\hline $6 \mathrm{H}-1,20-22.5$ & 40.51 & 596 & $\mathrm{CN} 12 \mathrm{~b}$ \\
\hline $6 \mathrm{H}-1,40-42.5$ & 40.71 & 803 & $\mathrm{CN} 12 \mathrm{~b}$ \\
\hline $6 \mathrm{H}-1,60-62.5$ & 40.91 & 586 & $\mathrm{CN} 12 \mathrm{~b}$ \\
\hline $6 \mathrm{H}-1,80-82.5$ & 41.11 & 1050 & $\mathrm{CN} 12 \mathrm{~b}$ \\
\hline $6 \mathrm{H}-1,100-102.5$ & 41.31 & 1100 & upper CN12a \\
\hline $6 \mathrm{H}-1,120-122.5$ & 41.51 & 903 & upper $\mathrm{CN} 12 \mathrm{a}$ \\
\hline $6 \mathrm{H}-1,140-142.5$ & 41.71 & 522 & upper $\mathrm{CN} 12 \mathrm{a}$ \\
\hline $6 \mathrm{H}-2,0-2.5$ & 41.81 & 773 & upper $\mathrm{CN} 12 \mathrm{a}$ \\
\hline $6 \mathrm{H}-2,20-22.5$ & 42.01 & 457 & upper $\mathrm{CN} 12 \mathrm{a}$ \\
\hline $6 \mathrm{H}-2,40-42.5$ & 42.21 & 736 & upper $\mathrm{CN} 12 \mathrm{a}$ \\
\hline $6 \mathrm{H}-2,60-62.5$ & 42.41 & 657 & upper $\mathrm{CN} 12 \mathrm{a}$ \\
\hline $6 \mathrm{H}-2,80-82.5$ & 42.61 & 1042 & upper $\mathrm{CN} 12 \mathrm{a}$ \\
\hline $6 \mathrm{H}-2,100-102.5$ & 42.81 & 536 & upper $\mathrm{CN} 12 \mathrm{a}$ \\
\hline $6 \mathrm{H}-2,120-122.5$ & 43.01 & 394 & upper $\mathrm{CN} 12 \mathrm{a}$ \\
\hline $6 \mathrm{H}-2,140-142.5$ & 43.21 & 585 & upper $\mathrm{CN} 12 \mathrm{a}$ \\
\hline $6 \mathrm{H}-3,0-2.5$ & 43.31 & 580 & upper $\mathrm{CN} 12 \mathrm{a}$ \\
\hline $6 \mathrm{H}-3,20-22.5$ & 43.51 & 820 & upper $\mathrm{CN} 12 \mathrm{a}$ \\
\hline $6 \mathrm{H}-3,40-42.5$ & 43.71 & 634 & upper CN12a \\
\hline $6 \mathrm{H}-3,60-62.5$ & 43.91 & 829 & upper $\mathrm{CN} 12 \mathrm{a}$ \\
\hline $6 \mathrm{H}-3,80-82.5$ & 44.11 & 681 & upper $\mathrm{CN} 12 \mathrm{a}$ \\
\hline $6 \mathrm{H}-3,100-102.5$ & 44.31 & 501 & upper CN12a \\
\hline $6 \mathrm{H}-3,120-122.5$ & 44.51 & 485 & upper $\mathrm{CN} 12 \mathrm{a}$ \\
\hline $6 \mathrm{H}-3,140-142.5$ & 44.71 & 745 & upper CN12a \\
\hline $6 \mathrm{H}-4,0-2.5$ & 44.81 & 611 & upper $\mathrm{CN} 12 \mathrm{a}$ \\
\hline $6 \mathrm{H}-4,20-22.5$ & 45.01 & 926 & upper $\mathrm{CN} 12 \mathrm{a}$ \\
\hline $6 \mathrm{H}-4,40-42.5$ & 45.21 & 712 & upper CN12a \\
\hline $6 \mathrm{H}-4,60-62.5$ & 45.41 & 823 & upper $\mathrm{CN} 12 \mathrm{a}$ \\
\hline $6 \mathrm{H}-4,80-82.5$ & 45.61 & 623 & upper $\mathrm{CN} 12 \mathrm{a}$ \\
\hline $6 \mathrm{H}-4,100-102.5$ & 45.81 & 682 & upper $\mathrm{CN} 12 \mathrm{a}$ \\
\hline $6 \mathrm{H}-4,120-122.5$ & 46.01 & 400 & upper $\mathrm{CN} 12 \mathrm{a}$ \\
\hline $6 \mathrm{H}-4,140-142.5$ & 46.21 & 641 & upper $\mathrm{CN} 12 \mathrm{a}$ \\
\hline $6 \mathrm{H}-5,0-2.5$ & 46.31 & 853 & lower $\mathrm{CN} 12 \mathrm{a}$ \\
\hline $6 \mathrm{H}-5,20-22.5$ & 46.51 & 432 & lower $\mathrm{CN} 12 \mathrm{a}$ \\
\hline $6 \mathrm{H}-5,40-42.5$ & 46.71 & 478 & lower CN12a \\
\hline $6 \mathrm{H}-5,60-62.5$ & 46.91 & 712 & lower $\mathrm{CN} 12 \mathrm{a}$ \\
\hline $6 \mathrm{H}-5,80-82.5$ & 47.11 & 901 & lower CN12a \\
\hline $6 \mathrm{H}-5,100-102.5$ & 47.31 & 932 & lower $\mathrm{CN} 12 \mathrm{a}$ \\
\hline $6 \mathrm{H}-5,120-122.5$ & 47.51 & 703 & lower $\mathrm{CN} 12 \mathrm{a}$ \\
\hline $6 \mathrm{H}-5,140-142.5$ & 47.71 & 616 & lower CN12a \\
\hline $6 \mathrm{H}-6,0-2.5$ & 47.81 & 658 & lower $\mathrm{CN} 12 \mathrm{a}$ \\
\hline $6 \mathrm{H}-6,20-22.5$ & 48.01 & 415 & lower CN12a \\
\hline $6 \mathrm{H}-6,40-42.5$ & 48.21 & 337 & lower $\mathrm{CN} 12 \mathrm{a}$ \\
\hline $6 \mathrm{H}-6,60-62.5$ & 48.41 & 553 & lower $\mathrm{CN} 12 \mathrm{a}$ \\
\hline $6 \mathrm{H}-6,80-82.5$ & 48.61 & 918 & lower CN12a \\
\hline $6 \mathrm{H}-6,100-102.5$ & 48.81 & 534 & lower CN12a \\
\hline $6 \mathrm{H}-6,120-122.5$ & 49.01 & 547 & upper $\mathrm{CN} 11 \mathrm{~b}$ \\
\hline $6 \mathrm{H}-6,140-142.5$ & 49.21 & 712 & upper $\mathrm{CN} 11 \mathrm{~b}$ \\
\hline $6 \mathrm{H}-7,0-2.5$ & 49.31 & 708 & upper $\mathrm{CN} 11 \mathrm{~b}$ \\
\hline $6 \mathrm{H}-7,20-22.5$ & 49.51 & 638 & upper $\mathrm{CN} 11 \mathrm{~b}$ \\
\hline $6 \mathrm{H}-7,40-42.5$ & 49.71 & 866 & upper $\mathrm{CN} 11 \mathrm{~b}$ \\
\hline $6 \mathrm{H}-7,60-62.5$ & 49.80 & 730 & upper $\mathrm{CN} 11 \mathrm{~b}$ \\
\hline
\end{tabular}

\begin{tabular}{|c|c|c|c|}
\hline $\begin{array}{l}\text { Core, section, } \\
\text { interval }(\mathrm{cm})\end{array}$ & $\begin{array}{l}\text { Depth } \\
\text { (mbsf) }\end{array}$ & F. profunda & Zone \\
\hline $7 \mathrm{H}-1,0-2.5$ & 49.81 & 931 & upper $\mathrm{CN} 11 \mathrm{~b}$ \\
\hline $7 \mathrm{H}-1,20-22.5$ & 50.01 & 440 & upper CN11b \\
\hline $7 \mathrm{H}-1,40-42.5$ & 50.21 & 797 & upper CN11b \\
\hline $7 \mathrm{H}-1,60-62.5$ & 50.41 & 623 & upper CN11b \\
\hline $7 \mathrm{H}-1,80-82.5$ & 50.61 & 838 & upper $\mathrm{CN} 11 \mathrm{~b}$ \\
\hline $7 \mathrm{H}-1,100-102.5$ & 50.81 & 618 & upper CN11b \\
\hline $7 \mathrm{H}-1,120-122.5$ & 51.01 & 610 & upper CN11b \\
\hline $7 \mathrm{H}-1,140-142.5$ & 51.21 & 720 & upper $\mathrm{CN} 11 \mathrm{~b}$ \\
\hline $7 \mathrm{H}-2,0-2.5$ & 51.31 & 1185 & upper CN11b \\
\hline $7 \mathrm{H}-2,20-22.5$ & 51.51 & 630 & upper $\mathrm{CN} 11 \mathrm{~b}$ \\
\hline $7 \mathrm{H}-2,40-42.5$ & 51.71 & 647 & upper CN11b \\
\hline $7 \mathrm{H}-2,60-62.5$ & 51.91 & 802 & upper CN11b \\
\hline $7 \mathrm{H}-2,80-82.5$ & 52.11 & 1264 & upper $\mathrm{CN} 11 \mathrm{~b}$ \\
\hline $7 \mathrm{H}-2,100-102.5$ & 52.31 & 1019 & upper CN11b \\
\hline $7 \mathrm{H}-2,120-122.5$ & 52.51 & 1072 & upper CN11b \\
\hline $7 \mathrm{H}-2,140-142.5$ & 52.71 & 986 & upper CN11b \\
\hline $7 \mathrm{H}-3,0-2.5$ & 52.81 & 1284 & upper CN11b \\
\hline $7 \mathrm{H}-3,20-22.5$ & 53.01 & 1144 & upper CN11b \\
\hline $7 \mathrm{H}-3,40-42.5$ & 53.21 & 1476 & upper CN11b \\
\hline $7 \mathrm{H}-3,60-62.5$ & 53.41 & 1020 & upper CN11b \\
\hline $7 \mathrm{H}-3,80-82.5$ & 53.61 & 977 & upper CN11b \\
\hline $7 \mathrm{H}-3,120-122.5$ & 54.01 & 1046 & upper CN11b \\
\hline $7 \mathrm{H}-3,140-142.5$ & 54.21 & 1192 & upper CN11b \\
\hline $7 \mathrm{H}-4,0-2.5$ & 54.31 & 1105 & upper CN11b \\
\hline $7 \mathrm{H}-4,20-22.5$ & 54.51 & 1020 & upper CN11b \\
\hline $7 \mathrm{H}-4,40-42.5$ & 54.71 & 1050 & upper $\mathrm{CN} 11 \mathrm{~b}$ \\
\hline $7 \mathrm{H}-4,60-62.5$ & 54.91 & 1376 & upper CN11b \\
\hline $7 \mathrm{H}-4,80-82.5$ & 55.11 & 1512 & upper CN11b \\
\hline $7 \mathrm{H}-4,100-102.5$ & 55.31 & 1104 & upper CN11b \\
\hline $7 \mathrm{H}-4,120-122.5$ & 55.51 & 1173 & upper CN11b \\
\hline $7 \mathrm{H}-4,140-142.5$ & 55.71 & 977 & upper CN11b \\
\hline $7 \mathrm{H}-5,0-2.5$ & 55.81 & 1113 & upper CN11b \\
\hline $7 \mathrm{H}-5,20-22.5$ & 56.01 & 1236 & upper CN11b \\
\hline $7 \mathrm{H}-5,40-42.5$ & 56.21 & 714 & upper CN11b \\
\hline $7 \mathrm{H}-5,60-62.5$ & 56.41 & 1125 & upper CN11b \\
\hline $7 \mathrm{H}-5,80-82.5$ & 56.61 & 1166 & upper CN11b \\
\hline $7 \mathrm{H}-5,100-102.5$ & 56.81 & 1401 & upper CN11b \\
\hline $7 \mathrm{H}-5,120-122.5$ & 57.01 & 1285 & upper CN11b \\
\hline $7 \mathrm{H}-5,140-142.5$ & 57.21 & 1055 & upper CN11b \\
\hline $7 \mathrm{H}-6,0-2.5$ & 57.31 & 631 & upper CN11b \\
\hline $7 \mathrm{H}-6,20-22.5$ & 57.51 & 1077 & upper CN11b \\
\hline $7 \mathrm{H}-6,40-42.5$ & 57.71 & 740 & upper CN11b \\
\hline $7 \mathrm{H}-6,60-62.5$ & 57.91 & 1114 & upper CN11b \\
\hline $7 \mathrm{H}-6,80-82.5$ & 58.11 & 982 & upper CN11b \\
\hline $7 \mathrm{H}-6,100-102.5$ & 58.31 & 1664 & upper CN11b \\
\hline $7 \mathrm{H}-6,120-122.5$ & 58.51 & 1545 & upper CN11b \\
\hline $7 \mathrm{H}-6,140-142.5$ & 58.71 & 1387 & upper CN11b \\
\hline $7 \mathrm{H}-7,0-2.5$ & 58.81 & 1280 & upper CN11b \\
\hline $7 \mathrm{H}-7,20-22.5$ & 59.01 & 1168 & upper CN11b \\
\hline $7 \mathrm{H}-7,40-42.5$ & 59.21 & 808 & upper CN11b \\
\hline $7 \mathrm{H}-7,60-62.5$ & 59.30 & 958 & upper CN11b \\
\hline $8 \mathrm{H}-1,0-2.5$ & 59.31 & 1240 & upper $\mathrm{CN} 11 \mathrm{~b}$ \\
\hline $8 \mathrm{H}-1,20-22.5$ & 59.51 & 1285 & upper CN11b \\
\hline $8 \mathrm{H}-1,40-42.5$ & 59.71 & 1034 & upper CN11b \\
\hline $8 \mathrm{H}-1,80-82.5$ & 60.11 & 1293 & upper CN11b \\
\hline $8 \mathrm{H}-1,100-102.5$ & 60.31 & 1048 & upper $\mathrm{CN} 11 \mathrm{~b}$ \\
\hline $8 \mathrm{H}-1,120-122.5$ & 60.51 & 576 & lower CN11 \\
\hline $8 \mathrm{H}-1,140-142.5$ & 60.71 & 973 & lower CN11 \\
\hline $8 \mathrm{H}-2,0-2.5$ & 60.81 & 930 & lower CN11 \\
\hline $8 \mathrm{H}-2,20-22.5$ & 61.01 & 996 & lower CN11 \\
\hline $8 \mathrm{H}-2,40-42.5$ & 61.21 & 1076 & lower CN11 \\
\hline $8 \mathrm{H}-2,60-62.5$ & 61.41 & 1108 & lower CN11 \\
\hline $8 \mathrm{H}-2,80-82.5$ & 61.61 & 1213 & lower CN11 \\
\hline $8 \mathrm{H}-2,100-102.5$ & 61.81 & 1136 & lower CN11 \\
\hline $8 \mathrm{H}-2,120-122.5$ & 62.01 & 754 & lower CN11 \\
\hline $8 \mathrm{H}-2,140-142.5$ & 62.21 & 921 & lower CN11 \\
\hline $8 \mathrm{H}-3,0-2.5$ & 62.31 & 742 & lower CN11 \\
\hline $8 \mathrm{H}-3,20-22.5$ & 62.51 & 1588 & lower CN11 \\
\hline $8 \mathrm{H}-3,40-42.5$ & 62.71 & 1150 & lower CN11 \\
\hline $8 \mathrm{H}-3,60-62.5$ & 62.91 & 1411 & lower CN11 \\
\hline $8 \mathrm{H}-3,80-82.5$ & 63.11 & 1322 & lower CN11 \\
\hline $8 \mathrm{H}-3,100-102.5$ & 63.31 & 1080 & lower CN11 \\
\hline $8 \mathrm{H}-3,120-122.5$ & 63.51 & 976 & lower CN11 \\
\hline $8 \mathrm{H}-3,140-142.5$ & 63.71 & 971 & lower CN11 \\
\hline $8 \mathrm{H}-4,0-2.5$ & 63.81 & 851 & lower CN11 \\
\hline $8 \mathrm{H}-4,20-22.5$ & 64.01 & 1135 & lower CN11 \\
\hline $8 \mathrm{H}-4,40-42.5$ & 64.21 & 432 & lower CN11 \\
\hline $8 \mathrm{H}-4,60-62.5$ & 64.41 & 1054 & lower CN11 \\
\hline $8 \mathrm{H}-4,80-82.5$ & 64.61 & 600 & lower CN11 \\
\hline $8 \mathrm{H}-4,100-102.5$ & 64.81 & 1230 & lower CN11 \\
\hline $8 \mathrm{H}-4,120-122.5$ & 65.01 & 733 & lower CN11 \\
\hline $8 \mathrm{H}-4,140-142.5$ & 65.21 & 415 & lower CN11 \\
\hline $8 \mathrm{H}-5,0-2.5$ & 65.31 & 645 & lower CN11 \\
\hline $8 \mathrm{H}-5,20-22.5$ & 65.51 & 950 & lower CN11 \\
\hline $8 \mathrm{H}-5,40-42.5$ & 65.71 & 954 & lower CN11 \\
\hline $8 \mathrm{H}-5,60-62.5$ & 65.91 & 1176 & lower CN11 \\
\hline $8 \mathrm{H}-5,80-82.5$ & 66.11 & 1187 & lower CN11 \\
\hline $8 \mathrm{H}-5,100-102.5$ & 66.31 & 755 & lower CN11 \\
\hline $8 \mathrm{H}-5,120-122.5$ & 66.51 & 600 & lower CN11 \\
\hline $8 \mathrm{H}-5,140-142.5$ & 66.71 & 940 & lower CN11 \\
\hline
\end{tabular}


Table 1 (continued).

\begin{tabular}{lccl}
\hline $\begin{array}{c}\text { Core, section, } \\
\text { interval (cm) }\end{array}$ & $\begin{array}{c}\text { Depth } \\
(\mathrm{mbsf})\end{array}$ & F. profunda & \multicolumn{1}{c}{ Zone } \\
\hline 159-959C- & & & \\
8H-6, 0-2.5 & 66.81 & 781 & lower CN11 \\
8H-6, 20-22.5 & 67.01 & 750 & lower CN11 \\
8H-6, 40-42.5 & 67.21 & 648 & lower CN11 \\
8H-6, 60-62.5 & 67.41 & 666 & lower CN11 \\
8H-6, 80-82.5 & 67.61 & 709 & lower CN11 \\
8H-6, 100-102.5 & 67.81 & 540 & lower CN11 \\
8H-6, 120-122.5 & 68.01 & 681 & lower CN11 \\
8H-6, 140-142.5 & 68.21 & 711 & lower CN11 \\
8H-7, 0-2.5 & 68.31 & 680 & lower CN11 \\
8H-7, 20-22.5 & 68.51 & 901 & lower CN11 \\
8H-7, 40-42.5 & 68.71 & 965 & CN 10d \\
8H-7, 60-62.5 & 68.80 & 967 & CN 10d \\
\hline
\end{tabular}

Pleistocene than the Pliocene, where warm-water Discoaster spp. are abundant.

The late Pliocene has the highest relative abundances of $F$. profunda, suggesting the deepest nutricline/thermocline depth, warm surface-water temperature, and low primary productivity in the upper euphotic water column because of the wind strength and divergence minima (Fig. 4). There is a significant change of both number (numbers $/ 20$ view fields) and relative abundances (\%) of $F$. profunda in the lower upper Pliocene (Sample 159-959C-6H-2, 80-82.5 cm; $42.61 \mathrm{mbsf}$ ) (Figs. 3, 4). Both the number and relative abundances of $F$. profunda gradually decrease from lower upper Pliocene (Sample 159-959C-6H-2, 80-82.5 cm; $42.61 \mathrm{mbsf}$ ) through the uppermost Pleistocene (Figs. 3, 4). This suggests that the nutricline/thermocline depth is gradually getting shallower from the lower upper Pliocene (Sample 159-959C-6H-2, 80-82.5 cm; $42.61 \mathrm{mbsf}$ ) through the uppermost Pleistocene. A decreasing trend of $F$. profunda from lower upper Pliocene (Sample 159-959C-6H-2, 80-82.5 cm; $42.61 \mathrm{mbsf}$ ) to the uppermost Pleistocene also suggests that the surface water cooled and productivity in the upper euphotic water column increased from the lower upper Pliocene through the uppermost Pleistocene (Fig. 4). The age of the sediments at $42.61 \mathrm{mbsf}$ is $\sim 2.82 \mathrm{Ma}$, by the assumption of a linear sediment accumulation rate between 2.73 and 3.65 $\mathrm{Ma}$. The sum of the relative abundances of warm-water species also shows climatic cooling starting at the lower upper Pliocene (upper part of Subzone 12a; near 2.82 Ma) (Fig. 6). Species richness (S) also shows a decreasing trend from the upper part of Subzone 12a (lower upper Pliocene) through the uppermost Pleistocene (Fig. 7).

Whitman and Berger (1993) reported climatic cooling at $2.87 \mathrm{Ma}$ based on the oxygen isotope record of foraminifers at the Pacific Site 586. The results of this study agrees well with that of the Whitman and Berger (1993).

The relative abundances of $F$. profunda decrease with decreased transparency in the euphotic water column at the northwestern margin of the Pacific (Ahagon et al., 1993). Gradual increases in water turbidity caused by increased particle and nutrient supplies from more exposed land areas, caused by lowering sea level, may be responsible for the decreasing trend of $F$. profunda starting from the lower upper Pliocene (Sample 159-959C-6H-2, 80-82.5 cm; 42.61 mbsf; $2.82 \mathrm{Ma}$ ) through uppermost Pleistocene. There is an abrupt fall of sea level shortly after $3 \mathrm{Ma}$ (Haq et al., 1987). As previously mentioned, the progressive productivity increase from lower upper Pliocene through uppermost Pleistocene matches well with the increased particles and nutrients at the same interval. Molfino and McIntyre (1990b) also reported that the rapid change of the relative abundance of $F$. profunda is associated with the boundary between high values of phosphate and low values of phosphate in the euphotic zone. Relatively greater sedimentation rate of the above time interval may support a great amount of terrigenous sediment input from nearby land areas (Shin et al., Chap. 39, this volume).
There is a significant shift in the relative abundance of $F$. profun$d a$ in the lowermost part of upper Pliocene (Sample 159-959C-6H-4, $80-82.5 \mathrm{~cm} ; 45.61 \mathrm{mbsf}$ ). The age of this depth is $\sim 3.03 \mathrm{Ma}$, based on the assumption of linear sediment accumulation rate between 2.73 and 3.65 Ma. Backman et al. (1986) reported changes in sediment accumulation rate in the North Atlantic at 3.2 Ma. There was a higher abyssal current velocity and an increase in the production of deep and bottom water at 3.2 Ma in the high-latitude North Atlantic (Backman et al., 1986). This study also shows sedimentation rate changes (Shin et al., Chap. 39, this volume), relative abundances of warm-water indicator calcareous nannofossils, and species diversity indices. Early late Pliocene (3.0 Ma) is a time of deepening of the carbonate compensation depth (CCD) to the present-day values (Rio et al., 1990). This CCD change corresponds with a climatic deterioration (Prell, 1984; Keigwin, 1982, 1987) and preceded the onset of the Northern Hemisphere glaciation that occurred at $2.5 \mathrm{Ma}$ (Shackleton et al., 1984). Shackleton et al. (1984) also reported that a considerable climatic variability existed from 2.4 to $3.5 \mathrm{Ma}$, before the initial glacial event. A significant climatic shift together with nutricline/thermocline depth change occurred between Sample 159-959C-6H-2, 80-82.5 cm (42.61 mbsf) and Sample 6H-4, 80-82.5 cm (45.61 mbsf). The duration of this 3-m core is $0.21 \mathrm{Ma}(3.03-2.82 \mathrm{Ma})$.

The relative abundances of $F$. profunda show a significant decreasing trend from the late late Pliocene (around 2.4 Ma) through the latest Pleistocene (Fig. 4). The percentages of warm-water indicator calcareous nannofossil species also show a decreasing trend from $\sim 2.4$ Ma through the Pleistocene (Fig. 6). Cool-water indicator calcareous nannofossils also show an increasing trend near the same time interval (Fig. 6). Lower abundances of Discoaster also occur at 2.4 Ma compared to the older sediment (Fig. 5). Species diversity indices $S$ and $H(S)$ also show decreasing trends from $\sim 2.4$ Ma to the core top (Fig. 7). All of these trends indicate climatic cooling from near 2.4 Ma to the younger sediments. First Pliocene glaciation is close to 2.4 Ma (Shackleton et al., 1984). Rapid cooling started at 2.4 $\mathrm{Ma}$ in the North Atlantic (Shackleton et al., 1984). Backman and Pestiaux (1987) also reported low Discoaster accumulation rates at 2.4 Ma in the North Atlantic, indicating cool surface temperature conditions.

The middle and upper Pleistocene (Zone CN15-CN14b) is characterized by the lowest relative abundances of $F$. profunda indicating the shallowest nutricline/thermocline depth and the coolest surfacewater temperatures caused by the maximum divergence and wind strength (Fig. 4). Pleistocene shallowing of the nutricline/thermocline were also reported in the Equatorial Atlantic as evidenced by the low relative abundances of $F$. profunda (Molfino and McIntyre, 1990a).

Species diversity of planktonic organisms shows an increasing trend in more stable marine conditions (Gibson, 1966; Gibson and Buzas, 1973). Watkins (1989) also observed that the higher $H(S)$ values of calcareous nannofossils indicate more stable surface-water conditions. Species diversity represents an inverse relationship to environmental stress (Sanders, 1968; Abele and Walters, 1979). The lower Pliocene is characterized by greater species diversity values. This suggests more stable surface-water conditions than the upper Pliocene and Pleistocene. Generally, the whole part of the core studied (Pliocene to Pleistocene) shows smaller fluctuations of the species diversity indices $S, H(S)$, and $E$, suggesting stable surface-water conditions during the deposition (Fig. 7).

\section{SUMMARY AND CONCLUSIONS}

The nutricline/thermocline depth fluctuated several times during the Pliocene to Pleistocene. The early Pliocene is characterized by a relatively shallow nutricline/thermocline and stable surface-water conditions compared to the late Pliocene. The late Pliocene has the 
Table 2. List of the discoasters (20 view fields), species diversity indices $(S, H[S], E)$, and sum of the relative abundances of cool- and warm-water indicator calcareous nannofossil species.

\begin{tabular}{|c|c|c|c|c|c|c|c|c|c|}
\hline Age & Nannofossil zone & $\begin{array}{l}\text { Core, section, } \\
\text { interval }(\mathrm{cm})\end{array}$ & $\begin{array}{l}\text { Depth } \\
\text { (mbsf) }\end{array}$ & $\begin{array}{c}\text { Discoasters } \\
(20 \text { view fields })\end{array}$ & $S$ & $H(S)$ & E & $\begin{array}{c}\text { Cool-water species } \\
(\%)\end{array}$ & $\begin{array}{c}\text { Warm-water species } \\
(\%)\end{array}$ \\
\hline & E. huxleyil & $1 \mathrm{H}-1,0-2.5$ & 0.01 & 0 & 9 & 1.81 & 0.68 & 29.44 & 42.17 \\
\hline late/middle & C. cristatus & $1 \mathrm{H}-1,80-82.5$ & 0.81 & 0 & 14 & 1.76 & 0.42 & 30.55 & 43.93 \\
\hline \multirow[t]{6}{*}{ Pleistocene } & (CN15/CN14b) & $2 \mathrm{H}-1,80-82.5$ & 3.11 & 0 & 8 & 1.1 & 0.38 & 29.04 & 8.32 \\
\hline & & $2 \mathrm{H}-2,80-82.5$ & 4.61 & 0 & 9 & 1.41 & 0.46 & 35.95 & 6.86 \\
\hline & & $2 \mathrm{H}-3,80-82.5$ & 6.11 & 0 & 7 & 1.08 & 0.42 & 16.72 & 22.45 \\
\hline & E. ovata & $2 \mathrm{H}-4,80-82.5$ & 7.61 & 0 & 10 & 1.34 & 0.38 & 8.09 & 43.63 \\
\hline & (CN14a) & $2 \mathrm{H}-5,80-82.5$ & 9.11 & 0 & 11 & 1.2 & 0.3 & 3.46 & 58.50 \\
\hline & & $2 \mathrm{H}-6,80-82.5$ & 10.61 & 0 & 11 & 1.36 & 0.35 & 4.80 & 34.50 \\
\hline \multirow{15}{*}{$\begin{array}{l}\text { early } \\
\text { Pleistocene }\end{array}$} & & $3 \mathrm{H}-1,80-82.5$ & 12.61 & 0 & 12 & 1.62 & 0.42 & 0.00 & 38.36 \\
\hline & & $3 \mathrm{H}-2,80-82.5$ & 14.11 & 0 & 10 & 0.89 & 0.24 & 0.00 & 17.59 \\
\hline & & $3 \mathrm{H}-3,80-82.5$ & 15.61 & 0 & 8 & 0.69 & 0.25 & 0.17 & 18.53 \\
\hline & G. caribbeanica & $3 \mathrm{H}-4,80-82.5$ & 17.11 & 0 & 14 & 1.7 & 0.39 & 4.27 & 25.30 \\
\hline & (CN13b) & $3 \mathrm{H}-5,80-82.5$ & 18.61 & 0 & 12 & 1.77 & 0.49 & 11.64 & 29.97 \\
\hline & & $3 \mathrm{H}-6,80-82.5$ & 20.11 & 0 & 14 & 1.44 & 0.3 & 5.81 & 40.68 \\
\hline & E. annula & $4 \mathrm{H}-1,80-82.5$ & 22.11 & 0 & 14 & 1.59 & 0.35 & 1.00 & 57.40 \\
\hline & (CN13a) & $4 \mathrm{H}-2,80-82.5$ & 23.61 & 0 & 16 & 1.62 & 0.32 & 1.01 & 58.87 \\
\hline & C. macintyrei & $4 \mathrm{H}-3,80-82.5$ & 25.11 & 8 & 17 & 1.65 & 0.31 & 0.40 & 45.80 \\
\hline & $(\mathrm{CN} 12 \mathrm{~d})$ & $4 \mathrm{H}-4,80-82.5$ & 26.61 & 9 & 18 & 1.24 & 0.19 & 1.40 & 66.80 \\
\hline & & $4 \mathrm{H}-5,80-82.5$ & 28.11 & 0 & 13 & 1.26 & 0.27 & 0.00 & 43.80 \\
\hline & & $4 \mathrm{H}-6,80-82.5$ & 29.61 & 0 & 15 & 1.41 & 0.27 & 0.40 & 48.60 \\
\hline & & $5 \mathrm{H}-1,80-82.5$ & 31.61 & 7 & 14 & 1.25 & 0.25 & 0.00 & 59.90 \\
\hline & D. pentaradiatus & $5 \mathrm{H}-2,80-82.5$ & 33.11 & 0 & 15 & 1.35 & 0.26 & 0.00 & 56.60 \\
\hline & (CN12c) & $5 \mathrm{H}-3,80-82.5$ & 34.61 & 1 & 13 & 1.21 & 0.26 & 0.00 & 58.80 \\
\hline \multirow{14}{*}{$\begin{array}{l}\text { late } \\
\text { Pliocene }\end{array}$} & & $5 \mathrm{H}-4,80-82.5$ & 36.11 & 6 & 13 & 0.98 & 0.2 & 0.00 & 71.14 \\
\hline & & $5 \mathrm{H}-5,80-82.5$ & 37.61 & 6 & 17 & 1.39 & 0.24 & 0.00 & 57.20 \\
\hline & & $5 \mathrm{H}-6,80-82.5$ & 39.11 & 12 & 16 & 1.25 & 0.22 & 0.00 & 64.40 \\
\hline & D. sur. $(\mathrm{CN} 12 \mathrm{~b})$ & $6 \mathrm{H}-1,80-82.5$ & 41.11 & 41 & 15 & 1.33 & 0.25 & 0.40 & 62.43 \\
\hline & D. tamalis & $6 \mathrm{H}-2,80-82.5$ & 42.61 & 21 & 18 & 1.03 & 0.16 & 0.20 & 77.10 \\
\hline & upper $\mathrm{CN} 12 \mathrm{a}$ & $6 \mathrm{H}-3,80-82.5$ & 44.11 & 23 & 17 & 1.66 & 0.31 & 0.20 & 44.54 \\
\hline & & $6 \mathrm{H}-4,80-82.5$ & 45.61 & 23 & 11 & 1.17 & 0.29 & 0.00 & 28.75 \\
\hline & D. tamalis & $6 \mathrm{H}-5,80-82.5$ & 47.11 & 35 & 15 & 1.35 & 0.26 & 0.00 & 39.58 \\
\hline & lower CN12a & $6 \mathrm{H}-6,80-82.5$ & 48.61 & 39 & 13 & 1.5 & 0.34 & 0.00 & 44.80 \\
\hline & & $7 \mathrm{H}-1,80-82.5$ & 50.61 & 29 & 18 & 1.92 & 0.38 & 0.00 & 42.40 \\
\hline & & $7 \mathrm{H}-2,80-82.5$ & 52.11 & 100 & 17 & 1.71 & 0.33 & 0.00 & 69.26 \\
\hline & D. asymmetricus & $7 \mathrm{H}-3,80-82.5$ & 53.61 & 59 & 16 & 2 & 0.46 & 0.20 & 58.00 \\
\hline & upper CN11b & $7 \mathrm{H}-4,80-82.5$ & 55.11 & 32 & 14 & 1.62 & 0.36 & 0.00 & 62.05 \\
\hline & & $7 \mathrm{H}-5,80-82.5$ & 56.61 & 27 & 16 & 1.78 & 0.37 & 0.00 & 60.60 \\
\hline \multirow{7}{*}{$\begin{array}{l}\text { early } \\
\text { Pliocene }\end{array}$} & & $7 \mathrm{H}-6,80-82.5$ & 58.11 & 42 & 17 & 1.79 & 0.35 & 0.20 & 62.00 \\
\hline & & $8 \mathrm{H}-1,80-82.5$ & 60.11 & 62 & 13 & 1.67 & 0.41 & 0.00 & 60.72 \\
\hline & & $8 \mathrm{H}-2,80-82.5$ & 61.61 & 136 & 15 & 1.69 & 0.36 & 0.00 & 50.80 \\
\hline & D. asymmetricus & $8 \mathrm{H}-3,80-82.5$ & 63.11 & 134 & 16 & 1.9 & 0.42 & 0.00 & 48.88 \\
\hline & lower CN11 & $8 \mathrm{H}-4,80-82.5$ & 64.61 & 63 & 19 & 2.16 & 0.46 & 0.20 & 44.60 \\
\hline & & $8 \mathrm{H}-5,80-82.5$ & 66.11 & 84 & 16 & 1.64 & 0.32 & 0.00 & 44.00 \\
\hline & & $8 \mathrm{H}-6,80-82.5$ & 67.61 & 107 & 16 & 1.94 & 0.43 & 0.00 & 33.80 \\
\hline
\end{tabular}

deepest nutricline/thermocline within the Pliocene to Pleistocene. A significant climatic and paleoceanographic shift occurred between 3.03 and $2.82 \mathrm{Ma}$ (early late Pliocene). The nutricline/thermocline depth becomes shallower from 2.82 Ma through the younger sediment. The surface-water temperature becomes cooler and surface water (upper euphotic water) primary productivity becomes higher starting from 2.82 Ma through the latest Pleistocene. The transition between deep and shallow nutricline/thermocline occurred at $3.03 \mathrm{Ma}$ (early late Pliocene). The nutricline/thermocline depth younger than 3.03 Ma is deeper than that of older than 3.03 Ma within Pliocene. The early Pleistocene shows great fluctuations of nutricline and thermocline depth. The middle and late Pleistocene is characterized by shallowest nutricline/thermocline.

\section{ACKNOWLEDGMENTS}

The study was supported by a grant from USSSAP (United States Science Support Program). I thank the Ocean Drilling Program and the Shipboard Scientists of ODP Leg 159 who made this study possible. My deep appreciation goes to David Watkins with whom I had many valuable discussions from which this work benefited. The paper was reviewed by Luc Beaufort and an anonymous reviewer. I gratefully acknowledge the time they spent, which materially improved the manuscript.

\section{REFERENCES}

Abele, L.G., and Walters, K., 1979. Marine benthic diversity: a critique and alternative explanation. J. Biogeogr., 6:115-126.

Ahagon, N., Tanaka, Y., and Ujiie, H., 1993. Florisphaera profunda, a possible nannoplankton indicator of late Quaternary changes in sea-water turbidity at the northwestern margin of the Pacific. Mar. Micropaleontol., 22:255-273.

Backman, J., and Pestiaux, P., 1987. Pliocene Discoaster abundance variations, Deep Sea Drilling Project Site 606: biochronology and paleoenvironmental implications. In Ruddiman, W.F., Kidd, R.B., Thomas, E., et al., Init. Repts. DSDP, 94 (Pt. 2): Washington (U.S. Govt. Printing Office), 903-910.

Backman, J., Pestiaux, P., Zimmerman, H., and Hermelin, J.O.R., 1986. Palaeoclimatic and palaeoceanographic development in the Pliocene North Atlantic: Discoaster accumulation and coarse fraction data. In Summerhayes, C.P., and Shackleton, N.J. (Eds.), North Atlantic Palaeoceanography. Geol. Soc. Spec. Publ. London, 21:231-241.

Backman, J., and Shackleton, N.J., 1983. Quantitative biochronology of Pliocene and early Pleistocene calcareous nannofossils from the Atlantic, Indian and Pacific oceans. Mar. Micropaleontol., 8:141-170.

Beaufort, L., 1991. Adaptation of the random settling method for quantitative studies of calcareous nannofossils. Micropaleontology, 34:415418.

Berggren, W.A., Hilgen, F.J., Langereis, C.G., Kent, D.V., Obradovich, J.D., Raffi, I., Raymo, M.E., and Shackleton, N.J., 1995. Late Neogene chronology: new perspectives in high-resolution stratigraphy. Geol. Soc. Am. Bull., 107:1272-1287.

Berggren, W.A., Kent, D.V., and Van Couvering, J.A., 1985. The Neogene, Part 2. Neogene geochronology and chronostratigraphy. In Snelling, N.J. 
(Ed.), The Chronology of the Geological Record. Geol. Soc. London Mem., 10:211-260.

Bukry, D., 1971. Discoaster evolutionary trends. Micropaleontology, 17:4352.

1978. Cenozoic coccolith and silicoflagellate stratigraphy, offshore northwest Africa, Deep Sea Drilling Project Leg 41. In Lancelot, Y., Seibold, E., et al., Init. Repts. DSDP, 41: Washington (U.S. Govt. Printing Office), 689-707.

Buzas, M.A., and Gibson, T., 1969. Species diversity: benthic foraminifera in western North Atlantic. Science, 163:72-75.

Chepstow-Lusty, A., Backman, J., and Shackleton, N.J., 1989. Comparison of upper Pliocene Discoaster abundance variations from North Atlantic Sites 552, 607, 658, 659 and 662: further evidence for marine plankton responding to orbital forcing. In Ruddiman, W.F., Sarnthein, M., et al., Proc. ODP, Sci. Results, 108: College Station, TX (Ocean Drilling Program), 121-141.

Decima, F.P., Medizza, F., and Todesco, L., 1978. Southeastern Atlantic Leg 40 calcareous nannofossils. In Bolli, H.M., Ryan, W.B.F., et al, Init. Repts. DSDP, 41: Washington (U.S. Govt. Printing Office), 571-634.

Ehrendorfer, T.W., 1993. Late Cretaceous (Maastrichtian) calcareous nannoplankton biogeography with emphasis on events immediately proceeding the Cretaceous/Paleocene boundary [Ph.D. dissert.]. Woods Hole Oceanographic Inst., Massachusetts Inst. of Technology.

Gibson, L.B., 1966. Some unifying characteristics of species diversity. Contrib. Cushman Found. Foraminifer Res., 17:117-124.

Gibson, T.G., and Buzas, M.A., 1973. Species diversity: pattern in Modern and Miocene Foraminifera of the eastern margin of North America. Geol. Soc. Am. Bull., 84:217-238.

Haq, B., 1971. Paleogene calcareous nannoflora, part IV. Paleogene nannoplankton biostratigraphy and evolutionary rates in Cenozoic calcareous nannoplankton. Stockholm Contrib. Geol., 25:129-158.

Haq, B.U., Hardenbol, J., and Vail, P.R., 1987. Chronology of fluctuating sea levels since the Triassic. Science, 235:1156-1167.

Haq, B.U., and Lohmann, G.P., 1976. Early Cenozoic calcareous nannoplankton biogeography of the Atlantic Ocean. Mar. Micropaleontol., 1:119-194.

Haq, B.U., Lohmann, G.P., and Wise, S.W., 1977. Calcareous nannoplankton biogeography and its paleoclimatic implications: Cenozoic of the Falkland Plateau (DSDP Leg 36) and Miocene of the North Atlantic Ocean. In Barker, P.F., Dalziel, I.W.D., et al., Init. Repts. DSDP, 36: Washington (U.S. Govt. Printing Office), 745-759.

Honjo, S., 1977. Biogeography and provincialism of living coccolithophorids in the Pacific Ocean. In Ramsay, A.T.S. (Ed.), Oceanic Micropaleontology: New York (Academic Press), 951-972.

Honjo, S., and Okada, H., 1974. Community structure of coccolithophores in the photic layer of the mid-Pacific. Micropaleontology, 20:209-230.

Huber, B.T., and Watkins, D.K., 1992. Biogeography of Campanian-Maastrichtian calcareous plankton in the region of the Southern Ocean: paleogeographic and paleoclimatic implications. $n$ Kennett, J.P., and Warnke, D.A. (Eds.), The Antarctic Paleoenvironment: A Perspective on Global Change. Am. Geophys. Union, Antarct. Res. Ser., 56:31-60.

Keigwin, L.D., 1987. Pliocene stable-isotope record of Deep Sea Drilling Project Site 606: sequential events of ${ }^{18} \mathrm{O}$ enrichment beginning at 3.1 Ma. In Ruddiman, W.F., Kidd, R.B., Thomas, E., et al., Init. Repts. DSDP, 94 (Pt. 2): Washington (U.S. Govt. Printing Office), 911-920.

Keigwin, L.D., Jr., 1982. Stable isotope stratigraphy and paleoceanography of Sites 502 and 503. In Prell, W.L., Gardner, J.V., et al., Init. Repts. DSDP, 68: Washington (U.S. Govt. Printing Office), 445-453.

Lohmann, G.P., and Carlson, J.J., 1981. Oceanographic significance of Pacific late Miocene calcareous nannoplankton. Mar. Micropaleontol., 6:553-579.

Manivit, H., 1989. Calcareous nannofossil biostratigraphy of Leg 108 sediments. In Ruddiman, W., Sarnthein, M., et al., Proc. ODP, Sci. Results, 108: College Station, TX (Ocean Drilling Program), 35-69.

McIntyre, A., Bé, A.W.H., and Roche, M.B., 1970. Modern Pacific coccolithophorida: a paleontological thermometer. Trans. N.Y. Acad. Sci., 32:720-731.

Molfino, B., and McIntyre, A., 1990a. Nutricline variations in the equatorial Atlantic coincident with the Younger Dryas. Paleoceanography, 5:9971008.

, 1990b. Precessional forcing of nutricline dynamics in the Equatorial Atlantic. Science, 249:766-769.
Okada, H., 1984. Modern nannofossil assemblages in sediments of coastal and marginal seas along the western Pacific ocean. Utrecht Micropaleontol. Bull., 30:171-187.

, 1990. Quaternary and Paleogene calcareous nannofossils, Leg 115. In Duncan, R.A., Backman, J., Peterson, L.C., et al., Proc. ODP, Sci. Results, 115: College Station, TX (Ocean Drilling Program), 129-174.

Okada, H., and Bukry, D., 1980. Supplementary modification and introduction of code numbers to the low-latitude coccolith biostratigraphic zonation (Bukry, 1973; 1975). Mar. Micropaleontol., 5:321-325.

Okada, H., and Honjo, S., 1973. The distribution of oceanic coccolithophorids in the Pacific. Deep-Sea Res. Part A, 20:355-374.

, 1975. Distribution of coccolithophores in marginal seas along the western Pacific Ocean and in the Red Sea. Mar. Biol., 31:271-285.

Okada, H., and McIntyre, A., 1977. Modern coccolithophores of the Pacific and North Atlantic Oceans. Micropaleontology, 23:1-55

, 1979. Seasonal distribution of the modern Coccolithophores in the western North Atlantic Ocean. Mar. Biol., 54:319-328.

Prell, W.L., 1984. Covariance patterns of foraminiferal $\delta^{18} \mathrm{O}$ : an evaluation of Pliocene ice volume changes near 3.2 million years ago. Science, 226:692-694.

Reid, F.M.H., 1980. Coccolithophorids of the North pacific Central Gyre with notes on their vertical and seasonal distribution. Micropaleontology, 26:151-176.

Rio, D., Fornaciari, E., and Raffi, I., 1990. Late Oligocene through early Pleistocene calcareous nannofossils from western equatorial Indian Ocean (Leg 115). In Duncan, R.A., Backman, J., Peterson, L.C., et al., Proc. ODP, Sci. Results, 115: College Station, TX (Ocean Drilling Program), 175-235.

Roth, P.H., 1973. Calcareous nannofossils-Leg 17, Deep Sea Drilling Project. In Winterer, E.L., Ewing, J.I., et al., Init. Repts. DSDP, 17: Washington (U.S. Govt. Printing Office), 695-795.

Sanders, H.L., 1968. Marine benthic diversity: a comparative study. Am. Nat., 102:243-282.

Schneidermann, N., 1977. Selective dissolution of Recent coccoliths in the Atlantic Ocean. In Ramsay, A.T.S. (Ed.), Oceanic Micropaleontology: New York (Academic Press), 1009-1053.

Shackleton, N.J., Backman, J., Zimmerman, H., Kent, D.V., Hall, M.A., Roberts, D.G., Schnitker, D., Baldauf, J.G., Desprairies, A., Homrighausen, R., Huddlestun, P., Keene, J.B., Kaltenback, A.J., Krumsiek, K.A.O., Morton, A.C., Murray, J.W., and Westberg-Smith, J., 1984. Oxygen isotope calibration of the onset of ice-rafting and history of glaciation in the North Atlantic region. Nature, 307:620-623.

Shipboard Scientific Party, 1996. Site 959. In Mascle, J., Lohmann, G.P. Clift, P.D., et al., Proc. ODP, Init. Repts., 159: College Station, TX (Ocean Drilling Program), 65-150.

Spaulding, S., 1991. Neogene nannofossil biostratigraphy of Sites 723 through 730, Oman Continental Margin, northwestern Arabian Sea. In Prell, W.L., Niitsuma, N., et al., Proc. ODP, Sci. Results, 117: College Station, TX (Ocean Drilling Program), 5-36.

Watkins, D.K., 1989. Nannoplankton productivity fluctuations and rhythmically-bedded pelagic carbonates of the Greenhorn Limestones (Upper Cretaceous). Palaeogeogr., Palaeoclimatol., Palaeoecol., 74:75-86.

Wei, W., 1988. A new technique for preparing quantitative nannofossil slides. J. Paleontol., 62:472-473.

Whitman, J.M., and Berger, W.H., 1993. Pliocene-Pleistocene carbon isotope record, Site 856, Ontong Java Plateau. In Berger, W.H., Kroenke, L.W., Mayer, L.A., et al., Proc. ODP, Sci. Results, 130: College Station, TX (Ocean Drilling Program), 333-348.

Winter, A., Reiss, Z., and Luz, B., 1979. Distribution of living coccolithophore assemblages in the Gulf of Elat (Aqaba). Mar. Micropaleontol., 4:197-223.

Wise, S.H., 1988. Mesozoic-Cenozoic history of calcareous nannofossils in the region of the Southern Ocean. Palaeogeogr., Palaeoclimatol., Palaeoecol., 67:157-179.

Date of initial receipt: 19 September 1996

Date of acceptance: 29 July 1997

Ms 159SR-046 


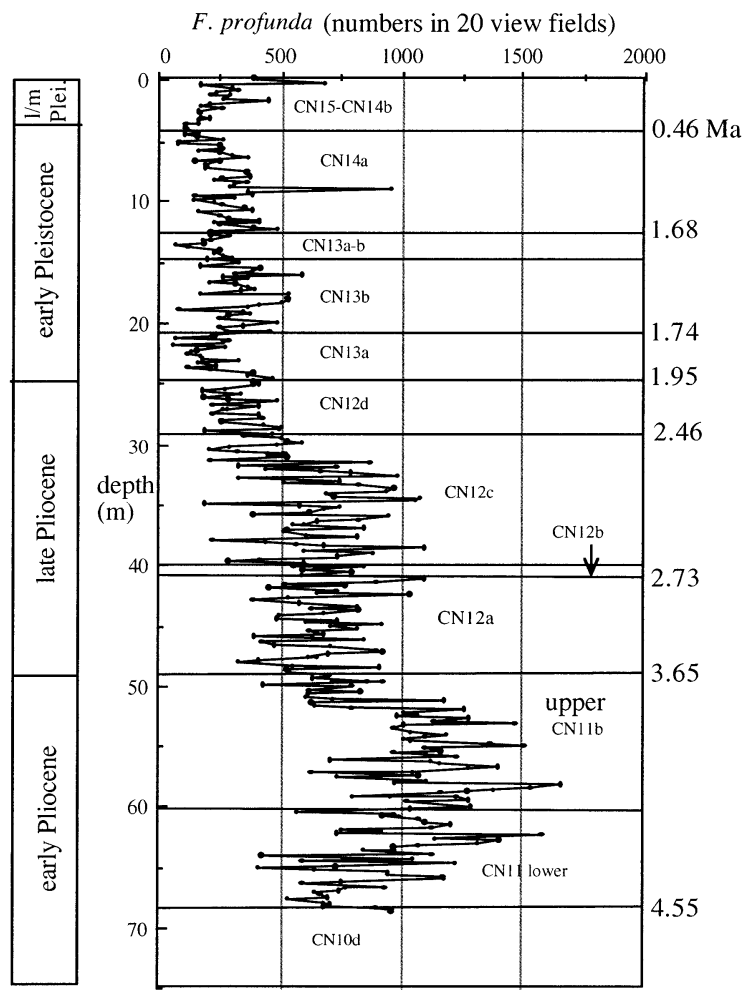

Figure 2. Depth-distribution patterns of calcareous nannofossil species $F$. profunda in 20 view fields at 20-cm intervals (magnification 1000×).

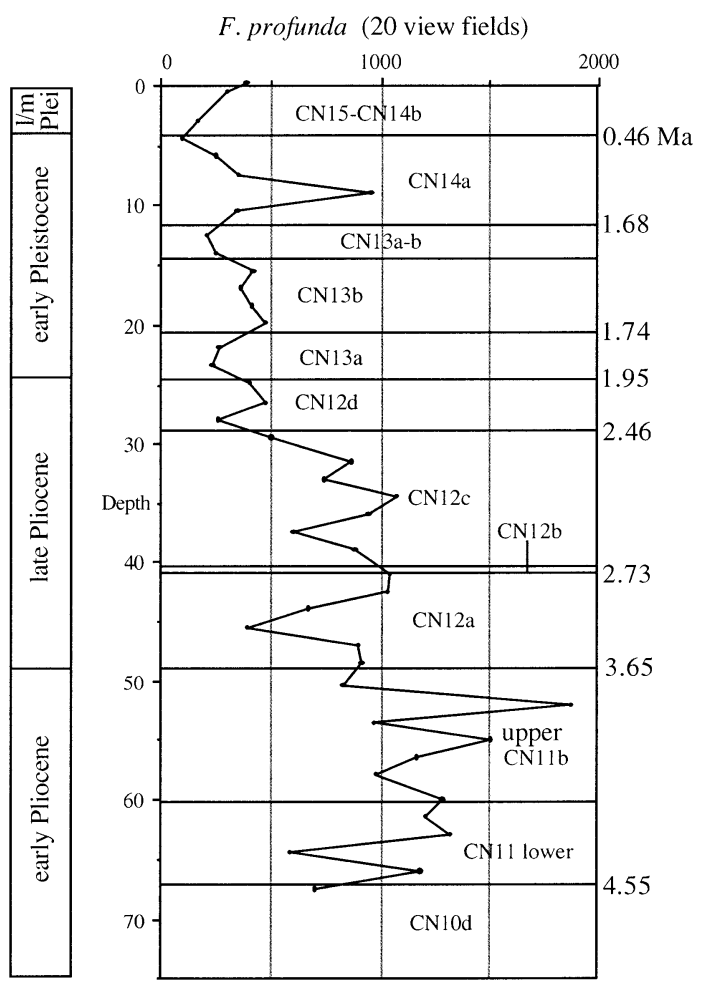

Figure 3. Depth-distribution patterns of the numbers (20 view fields) of $F$. profunda in each section.

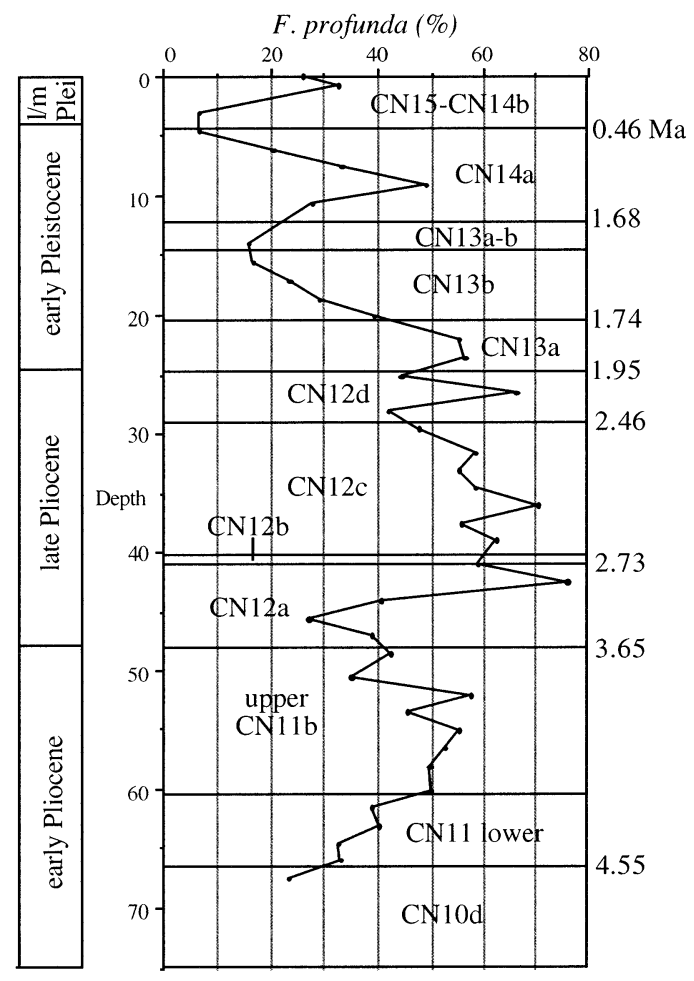

Figure 4. Depth-distribution patterns of the relative percentages of F. profunda in each section.

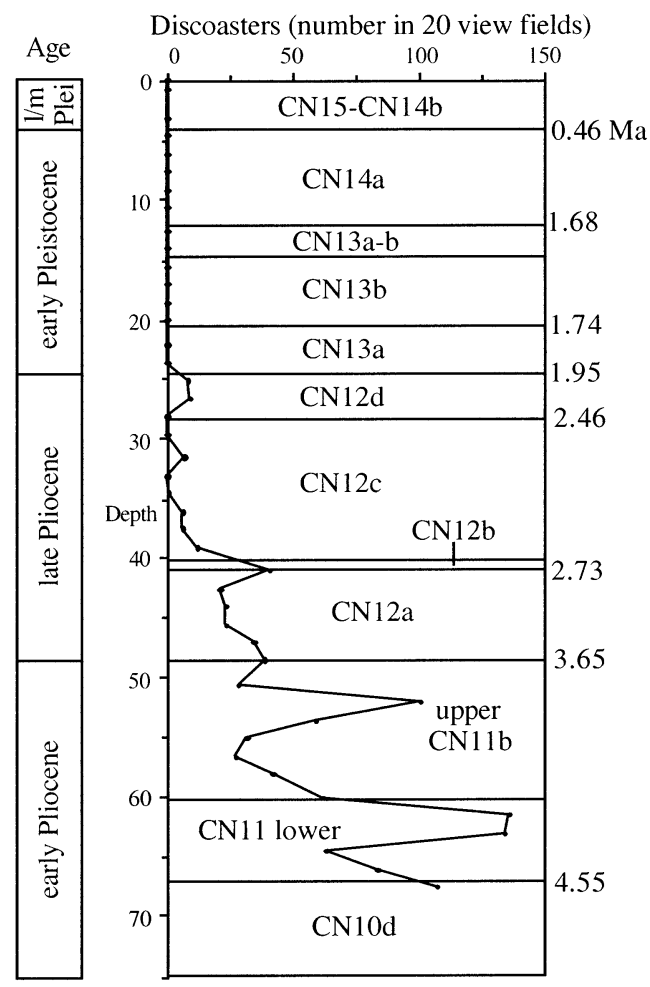

Figure 5. Depth-distribution patterns of the number of Discoasters in each section (magnification 1000×). 


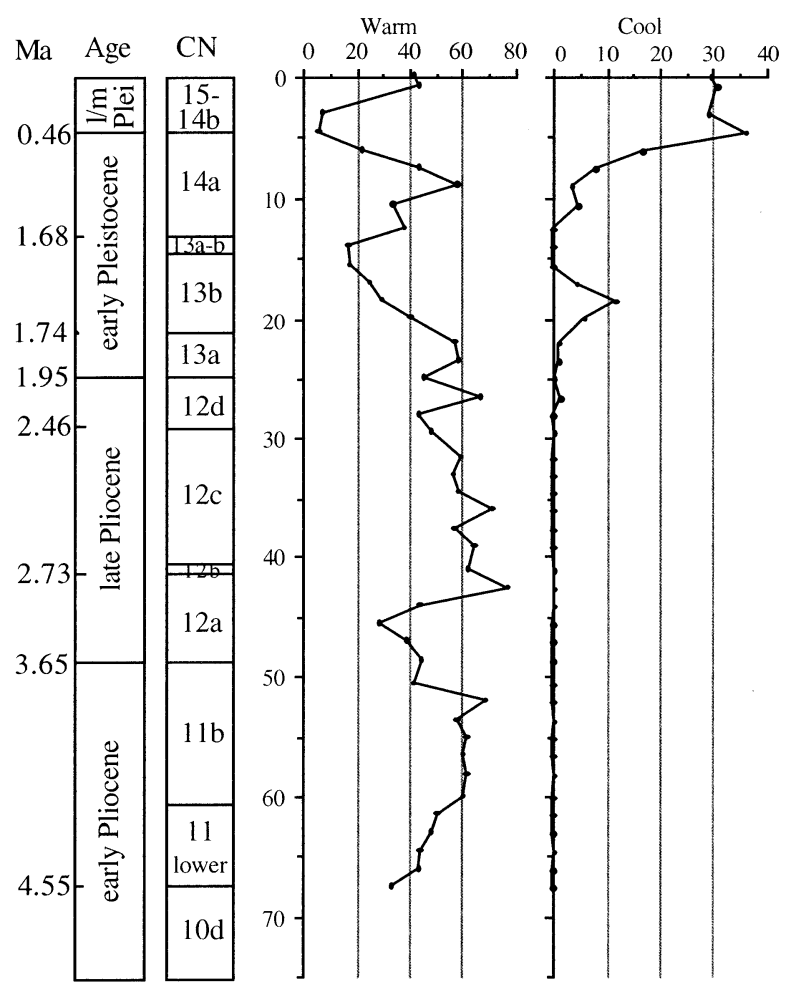

Figure 6. Relative abundances of warm- and cool-water indicator calcareous nannofossil species in each section.

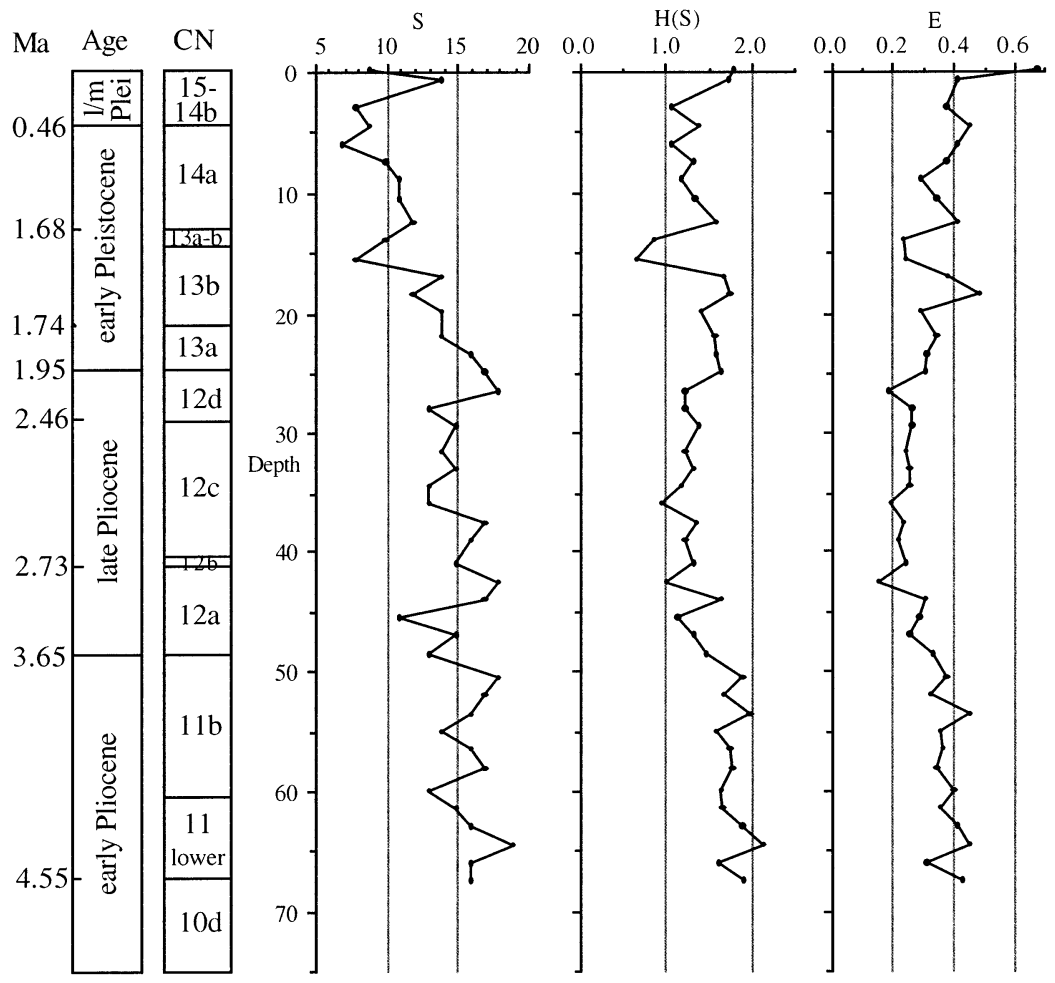

Figure 7. Species diversity indices $S, H(S)$, and $E$ in each section. 